\title{
THE BOCHNER-KODAIRA-NAKANO FORMULA FOR LINE BUNDLES ON CR MANIFOLDS WITH TRANSVERSAL CR $\mathbb{R}$-ACTION
}

\author{
HUAN WANG
}

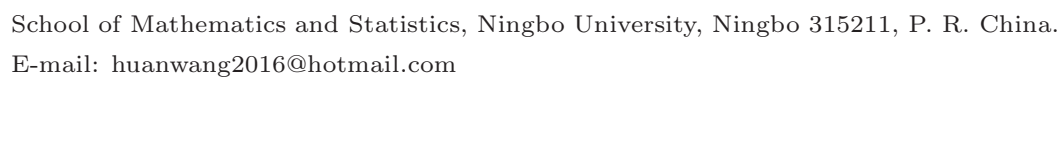

Abstract

We prove the generalized Kähler identities for line bundles on CR manifolds with transversal CR $\mathbb{R}$-action and reproduce the Bochner-Kodaira-Nakano formulas by the standard approach, in which the contribution from the curvature of line bundle and Levi form is explicitly expressed. The related results and various cohomology vanishing theorems are given.

\section{Introduction}

The Bochner-Kodaira-Nakano formula [2] plays an important role in complex geometry, which is related to some classical results like Kodaira embedding theorem and Riemann-Roch-Hirzebruch formula. The counterpart of these results are well established for CR manifold with transversal CR $S^{1}$-action [4, 1] and related recent progress [1] [12] [14] [10] [6]. However, the Bochner-Kodaira-Nakano formula seems absent in this context. In this note we follow the standard approach, namely, generalized Kähler identities, to reproduce the Bochner-Kodaira-Nakano formula on CR manifolds with transversal CR $\mathbb{R}$-action firstly obtained in [9]. The approach here exhibits explicitly how the term on the curvature of line bundle and Levi form appears. The key ingredient in the Bochner-Kodaira-Nakano formula [2] is the term $\left[\sqrt{-1} R^{E}, \Lambda\right]$, where $R^{E}$ is the curvature of holomorphic vector bundle and $\Lambda$ is the dual of Hermitian metric on complex manifolds. The new

Received May 15, 2021.

AMS Subject Classification: 32A50.

Key words and phrases: CR manifolds, Kohn Laplacian, Bochner-Kodaira-Nakano formula. 
figure of our formula (Theorem [1.2) is the term $\left[2 \mathscr{L} T+\sqrt{-1} R^{L}, \Lambda\right]$, which depends on the curvature of CR line bundle $\sqrt{-1} R^{L}$, the Levi form $\mathscr{L}$ and the uniquely determined global real vector field $T$ on CR manifolds.

We will work in the following setting. Let $X$ be an orientable CR manifold of dimension $2 n+1, n \geq 1, J$ is the complex structure and $\omega_{0}$ is a contact form, endowed with a smooth transversal CR $\mathbb{R}$-action on $X$ preserving $\omega_{0}$ and $J$. Let $\mathscr{L}=\frac{d \omega_{0}}{2 i}$ be the Levi form with respect to $\omega_{0}$. Let $T^{1,0} X$ and $T^{0,1} X$ be the bundles of tangent vectors of type $(1,0)$ and $(0,1)$, respectively. Since there is a transversal $\mathrm{CR} \mathbb{R}$-action, we have a decomposition $\mathbb{C} T X=T^{1,0} X \oplus T^{0,1} X \oplus \mathbb{C} T$, where $T$ is the infinitesimal generator of the $\mathbb{R}$-action. We choose the $\mathbb{R}$-invariant contact form $\omega_{0}$ such that $\omega_{0}(T)=1$. Suppose there exists an $\mathbb{R}$-invariant Hermitian metric $\langle\cdot \mid \cdot\rangle$ on $\mathbb{C} T X$ such that

$$
T^{1,0} X \perp T^{0,1} X, T \perp\left(T^{1,0} X \oplus T^{0,1} X\right),\langle T \mid T\rangle=1 .
$$

Moreover, we denote by $\Theta_{X}$ its fundamental $(1,1)$-form given by $\Theta_{X}(u, \bar{v})=$ $\sqrt{-1}\langle u \mid v\rangle$ for $u, v \in T^{1,0} X$. Let $\Theta_{X} \wedge$. be the Lefschetz operator and $\Lambda=i\left(\Theta_{X}\right)$ its adjoint with respect to the Hermitian metric $\langle\cdot \mid \cdot\rangle$. The Hermitian torsion of $\Theta_{X}$ is defined by $\mathcal{T}:=\left[\Lambda, \partial_{b} \Theta_{X}\right]$. Let $K_{X}:=\operatorname{det}\left(T^{1,0 *} X\right)$ be the determinant of the dual bundle of $T^{1,0} X$. Let $L$ be a $\mathbb{R}$-equivariant CR line bundle over $X$ with a $\mathbb{R}$-invariant Hermitian metric $h^{L}$ on $L$. Let $R^{L}$ be the curvature of $L$ induced by $h^{L}$. For $p, q \in \mathbb{N}_{0}$, let $\Omega^{p, q}(X, L)$ be the space of smooth $(p, q)$ forms of $X$ with values in $L$ and let $\Omega^{\bullet \bullet \bullet}(X, L):=$ $\oplus_{p, q \in \mathbb{N}_{0}} \Omega^{p, q}(X, L)$. Let $\Omega_{c}^{p, q}(X, L)$ be the subspace of $\Omega^{p, q}(X, L)$ whose elements have compact support in $X$ and let $\Omega_{c}^{\bullet, \bullet}(X, L):=\oplus_{p, q \in \mathbb{N}_{0}} \Omega_{c}^{p, q}(X, L)$. For $p, q \in \mathbb{N}_{0}$, let $\bar{\partial}_{b}^{L}: \Omega^{p, q}(X, L) \rightarrow \Omega^{p, q+1}(X, L)$ be the tangential CauchyRiemann operator with values in $L$. Let $(\cdot \mid \cdot)_{L}$ be the $L^{2}$ inner product on $\Omega_{c}^{\bullet \bullet \bullet}(X, L)$ induced by $\langle\cdot \mid \cdot\rangle$ and $h^{L}$. Let $\bar{\partial}_{b}^{L *}: \Omega^{p, q+1}(X, L) \rightarrow \Omega^{p, q}(X, L)$ be the formal adjoint of $\bar{\partial}_{b}^{L}$ with respect to $(\cdot \mid \cdot)_{L}$. The Kohn Laplacian with respect to $L$ is given by

$$
\square_{b}^{L}:=\bar{\partial}_{b}^{L} \bar{\partial}_{b}^{L *}+\bar{\partial}_{b}^{L *} \bar{\partial}_{b}^{L}
$$

Let $\nabla_{b}^{L}: \Omega^{\bullet \bullet \bullet}(X, L) \rightarrow \Omega^{\bullet \bullet \bullet}(X, L)$ be the connection on $L$ induced by $h^{L}$, which splits into $\nabla_{b}^{L}=\left(\nabla_{b}^{L}\right)^{1,0}+\bar{\partial}_{b}^{L}$. Let $\left(\nabla_{b}^{L}\right)^{1,0 *}$ be the adjoint of $\left(\nabla_{b}^{L}\right)^{1,0}$ 
with respect to $(\cdot \mid \cdot)_{L}$. The anti-Kohn Laplacian with respect to $L$ is given by

$$
\square_{b}^{L}:=\left(\nabla_{b}^{L}\right)^{1,0}\left(\nabla_{b}^{L}\right)^{1,0 *}+\left(\nabla_{b}^{L}\right)^{1,0 *}\left(\nabla_{b}^{L}\right)^{1,0} .
$$

Now we formulate the main result.

Proposition 1.1 (Generalized Kähler identities for CR line bundle). Let $X$ be a CR manifold with a transversal $C R \mathbb{R}$-action. Let $\Theta_{X}$ be an $\mathbb{R}$-invariant Hermitian metric on $X$. Let $L$ be a $\mathbb{R}$-equivariant $C R$ line bundle over $X$ with a $\mathbb{R}$-invariant Hermitian metric $h^{L}$. With the notations used above, we have on $\Omega^{\bullet \bullet \bullet}(X, L)$,

$$
\begin{aligned}
{\left[\bar{\partial}_{b}^{L *}, \Theta_{X} \wedge\right] } & =\sqrt{-1}\left(\left(\nabla_{b}^{L}\right)^{1,0}+\mathcal{T}\right), \\
{\left[\left(\nabla_{b}^{L}\right)^{1,0 *}, \Theta_{X} \wedge\right] } & =-\sqrt{-1}\left(\bar{\partial}_{b}^{L}+\overline{\mathcal{T}}\right), \\
{\left[\Lambda, \bar{\partial}_{b}^{L}\right] } & =-\sqrt{-1}\left(\left(\nabla_{b}^{L}\right)^{1,0 *}+\mathcal{T}^{*}\right), \\
{\left[\Lambda,\left(\nabla_{b}^{L}\right)^{1,0}\right] } & =\sqrt{-1}\left(\bar{\partial}_{b}^{L *}+\overline{\mathcal{T}}^{*}\right) .
\end{aligned}
$$

We obtain the following Bochner-Kodaira-Nakano formula on CR manifolds with $\mathbb{R}$-action $[9$, Theorem 3.5] via the generalized Kähler identities.

Theorem 1.2. Let $X$ be a $C R$ manifold with a transversal $C R \mathbb{R}$-action. Let $\Theta_{X}$ be an $\mathbb{R}$-invariant Hermitian metric on $X$. Let $L$ be a $\mathbb{R}$-equivariant $C R$ line bundle over $X$ with a $\mathbb{R}$-invariant Hermitian metric $h^{L}$. With the notations used above, we have on $\Omega^{\bullet \bullet \bullet}(X, L)$,

$$
\square_{b}^{L}=\bar{\square}_{b}^{L}+\left[2 \mathscr{L} T+\sqrt{-1} R^{L}, \Lambda\right]+\left[\left(\nabla_{b}^{L}\right)^{1,0}, \mathcal{T}^{*}\right]-\left[\bar{\partial}_{b}^{L}, \overline{\mathcal{T}}^{*}\right] .
$$

If $X$ is Kähler, i.e., $d \Theta_{X}=0$, then

$$
\square_{b}^{L}=\bar{\square}_{b}^{L}+\left[2 \mathscr{L} T+\sqrt{-1} R^{L}, \Lambda\right] .
$$

If $X$ is Levi flat, i.e., $\mathscr{L}=0$, then

$$
\square_{b}^{L}=\bar{\square}_{b}^{L}+\left[\sqrt{-1} R^{L}, \Lambda\right]+\left[\left(\nabla_{b}^{L}\right)^{1,0}, \mathcal{T}^{*}\right]-\left[\bar{\partial}_{b}^{L}, \overline{\mathcal{T}}^{*}\right] .
$$

If $\left(L, h^{L}\right)$ is flat, i.e., $R^{L}=0$, then

$$
\square_{b}^{L}=\bar{\square}_{b}^{L}+[2 \mathscr{L} T, \Lambda]+\left[\left(\nabla_{b}^{L}\right)^{1,0}, \mathcal{T}^{*}\right]-\left[\bar{\partial}_{b}^{L}, \overline{\mathcal{T}}^{*}\right] .
$$


If $\left(L, h^{L}\right)$ is trivial, i.e., $L=X \times \mathbb{C}$ and $|(x, 1)|_{h^{L}}=1$, then

$$
\square_{b}=\bar{\square}_{b}+[2 \mathscr{L} T, \Lambda]+\left[\partial_{b}, \mathcal{T}^{*}\right]-\left[\bar{\partial}_{b}, \overline{\mathcal{T}}^{*}\right] .
$$

In order to eliminate the first order error term $\left[\left(\nabla_{b}^{L}\right)^{1,0}, \mathcal{T}^{*}\right]-\left[\bar{\partial}_{b}^{L}, \overline{\mathcal{T}}^{*}\right]$ in (1.2), we can also reformulate (1.2) as in [3, VII.1]:

Theorem 1.3. Under the hypothesis of Theorem 1.2 , on $\Omega^{\bullet \bullet \bullet}(X, L)$, we have

$$
\square_{b}^{L}=\bar{\square}_{b, \mathcal{T}}^{L}+\left[2 \mathscr{L} T+\sqrt{-1} R^{L}, \Lambda\right]+\mathcal{T}_{\Theta},
$$

where $\square_{b, \mathcal{T}}^{L}:=\left[\left(\nabla_{b}^{L}\right)^{1,0}+\mathcal{T},\left(\nabla_{b}^{L}\right)^{1,0 *}+\mathcal{T}^{*}\right]$ is a positive formally self-adjoint operator, and

$$
\mathcal{T}_{\Theta}:=\left[\Lambda,\left[\Lambda, \frac{\sqrt{-1}}{2} \partial_{b} \bar{\partial}_{b} \Theta_{X}\right]\right]-\left[\bar{\partial}_{b} \Theta_{X},\left(\bar{\partial}_{b} \Theta_{X}\right)^{*}\right]
$$

is an operator of order zero depending only on the torsion of Hermitian metric $\Theta_{X}$.

Remark 1.4. The above results hold both for transversal CR $\mathbb{R}$-action and $S^{1}$-action.

From now on we consider transversal CR $S^{1}$-action. For $r, q \in \mathbb{N}_{0}$ and $m \in \mathbb{Z}$, we set

$$
\Omega_{m}^{r, q}(X, L):=\left\{s \in \Omega_{m}^{r, q}(X, L):-\sqrt{-1} T s=m s\right\}
$$

and define the $m$-th Fourier component of Kohn-Rossi cohomology group with respect to $L$ by

$$
H_{m}^{r, q}(X, L):=\frac{\operatorname{Ker} \bar{\partial}_{b}^{L} \cap \Omega_{m}^{r, q}(X, L)}{\operatorname{Im} \bar{\partial}_{b}^{L} \cap \Omega_{m}^{r, q}(X, L)} .
$$

For simplicity, we set $H_{m}^{q}(X, L):=H_{m}^{0, q}(X, L)$. As corollaries of Theorem 1.2. we provide two basic vanishing theorems. It is clear that, from Theorem 1.2 one can obtain various generalization of them along Bochner techniques.

Corollary 1.5 (Nakano type vanishing theorem). Let $X$ be a compact $C R$ manifold of dimension $2 n+1$ with a transversal $C R S^{1}$-action and let $L$ be a $S^{1}$-equivariant $C R$ line bundle over $X$ with a $S^{1}$-invariant Hermitian metric 
$h^{L}$ on $L$. Let $m_{0} \in \mathbb{Z}$ such that $2 m_{0} \sqrt{-1} \mathscr{L}+\sqrt{-1} R^{L}>0$ on $X$. Then, for any $r, q \in \mathbb{N} \cup\{0\}$ satisfying $r+q>n$, we have

$$
H_{b, m_{0}}^{r, q}(X, L)=0
$$

In particular, $H_{b, m_{0}}^{q}\left(X, K_{X} \otimes L\right)=0$ for all $q>0$.

Corollary 1.6 (Kodaira-Serre type vanishing theorem). Let $X$ be a compact $C R$ manifold of dimension $2 n+1$ with a transversal $C R S^{1}$-action and let $L$ be a $S^{1}$-equivariant $C R$ line bundle over $X$ with a $S^{1}$-invariant Hermitian metric $h^{L}$ on L. Let $2 \sqrt{-1} \mathscr{L}+\sqrt{-1} R^{L}>0$ on $X$. Then, there exists $m_{0}>0$ such that for each $m>m_{0}$ and $q>0$,

$$
H_{b, m}^{q}\left(X, L^{m}\right)=0
$$

This paper is organized in the following way. In Sec. 2, we introduce the notations and the necessary facts. In Sec. 3, we prove Proposition 1.1, Theorem 1.2, 1.3 and the twisted version of CR Bochner-Kodaira-Nakano formula. In Sec. 4, we prove CR Nakano inequalities and Corollary 1.5, 1.6. In Sec. 5, further results and application are given.

\section{Preliminaries and Notations}

We use the following notations through this article: let $\mathbb{N}=\{1,2, \ldots\}$ be the set of natural numbers, $\mathbb{N}_{0}=\mathbb{N} \bigcup\{0\}, \mathbb{R}$ the set of real numbers. For $m \in \mathbb{N}$, let $x=\left(x_{1}, \ldots, x_{m}\right)$ be coordinates of $\mathbb{R}^{m}$. For $n \in \mathbb{N}$, let $z=\left(z_{1}, \ldots, z_{n}\right), z_{j}=x_{2 j-1}+\sqrt{-1} x_{2 j}, j=1, \ldots, n$, be coordinates of $\mathbb{C}^{n}$. We write

$$
\begin{array}{ccc}
d z_{j}=d x_{2 j-1}+\sqrt{-1} d x_{2 j}, & d \bar{z}_{j}=d x_{2 j-1}-\sqrt{-1} d x_{2 j}, \\
\frac{\partial}{\partial z_{j}}:=\frac{1}{2}\left(\frac{\partial}{\partial x_{2 j-1}}-\sqrt{-1} \frac{\partial}{\partial x_{2 j}}\right), & \frac{\partial}{\partial \bar{z}_{j}}:=\frac{1}{2}\left(\frac{\partial}{\partial x_{2 j-1}}+\sqrt{-1} \frac{\partial}{\partial x_{2 j}}\right) .
\end{array}
$$

For arbitrary $\mathbb{Z}_{2}$-graded vector space $V=V^{+} \oplus V^{-}$, the natural $\mathbb{Z}_{2}$-grading on $\operatorname{End}(V)$ is defined by $\operatorname{End}(V)^{+}=\operatorname{End}\left(V^{+}\right) \oplus \operatorname{End}\left(V^{-}\right)$, End $(V)^{-}=$ $\operatorname{Hom}\left(V^{+}, V^{-}\right) \oplus \operatorname{Hom}\left(V^{-}, V^{+}\right)$, and we set $\operatorname{deg} A=0$ for $A \in \operatorname{End}(V)^{+}$and 
$\operatorname{deg} A=1$ for $A \in \operatorname{End}(V)^{-}$. For $A, B \in \operatorname{End}(V)$, their supercommutator (or graded Lie bracket) is defined by

$$
[A, B]=A B-(-1)^{\operatorname{deg} A \cdot \operatorname{deg} B} B A .
$$

For $A, B, C \in$ End $(V)$, the Jacobi identity holds:

$$
\begin{aligned}
(-1)^{\operatorname{deg} A \cdot \operatorname{deg} C}[A,[B, C]] & +(-1)^{\operatorname{deg} B \cdot \operatorname{deg} A}[B,[C, A]] \\
& +(-1)^{\operatorname{deg} C \cdot \operatorname{deg} B}[C,[A, B]]=0 .
\end{aligned}
$$

\subsection{CR manifolds and Kohn Laplacian}

Let $X$ be a smooth paracompact manifold. Let $T X$ and $T^{*} X$ be the tangent bundle of $X$ and the cotangent bundle of $X$, respectively. The complexified tangent bundle of $X$ and the complexified cotangent bundle of $X$ are denoted by $\mathbb{C} T X$ and $\mathbb{C} T^{*} X$, respectively. We write $\langle\cdot, \cdot\rangle$ to denote the pointwise duality between $T X$ and $T^{*} X$ and extend $\langle\cdot, \cdot\rangle$ bilinearly to $\mathbb{C} T X \times \mathbb{C} T^{*} X$.

Let $X$ be an orientable paracompact smooth manifold of dimension $2 n+$ 1 with $n \geq 1$. Let $T^{1,0} X$ be a subbundle of rank $n$ of the complexified tangent bundle $\mathbb{C} T X$. Let $T^{0,1} X:=\overline{T^{1,0} X}$. Let $\mathscr{C}^{\infty}\left(X, T^{1,0} X\right)$ be the space of smooth sections of $T^{1,0} X$ on $X . T^{1,0} X$ is called a CR structure of $X$, if

$T^{1,0} X \cap T^{0,1} X=\{0\}, \quad\left[\mathscr{C}^{\infty}\left(X, T^{1,0} X\right), \mathscr{C}^{\infty}\left(X, T^{1,0} X\right)\right] \subset \mathscr{C}^{\infty}\left(X, T^{1,0} X\right)$.

$\left(X, T^{1,0} X\right)$ is called a $\mathrm{CR}$ manifold of dimension $2 n+1$, if $T^{1,0} X$ is a CR structure of $X$.

Now, let $\left(X, T^{1,0} X\right)$ be an orientable CR manifold of dimension $2 n+1$ with $n \geq 1$. Let $\langle\cdot \mid \cdot\rangle: \mathbb{C} T X \times \mathbb{C} T X \rightarrow \mathbb{C}$ be a smooth Hermitian inner product on $\mathbb{C} T X$ such that

$$
\langle u \mid v\rangle \in \mathbb{R} \quad \text { for } u, v \in T X, \quad\langle\alpha \mid \beta\rangle=0 \quad \text { for } \alpha \in T^{1,0} X, \beta \in T^{0,1} X .
$$

We call $\langle\cdot \mid \cdot\rangle$ a Hermitian metric on $X$. Moreover, let $\Theta_{X}$ be the fundamental $(1,1)$-form given by $\Theta_{X}(u, \bar{v})=\sqrt{-1}\langle u \mid v\rangle$ for $u, v \in T^{1,0} X$. The Hermitian norm is given by $|\cdot|:=\sqrt{\langle\cdot \mid \cdot\rangle}$. Until further notice, we fix a Hermitian metric $\langle\cdot \mid \cdot\rangle$ on $X$. 
For $p, q \in \mathbb{N}_{0}$, define $T^{p, q} X:=\left(\Lambda^{p} T^{1,0} X\right) \wedge\left(\Lambda^{q} T^{0,1} X\right)$ and let $T^{\bullet \bullet \bullet} X=$ $\bigoplus_{p, q \in \mathbb{N}_{0}} T^{p, q} X$. For $u \in \mathbb{C} T X$ and $\phi \in \mathbb{C} T^{*} X$, the pointwise duality is defined by $\langle u, \phi\rangle:=\phi(u)$. Let $T^{* 1,0} X \subset \mathbb{C} T^{*} X$ be the dual bundle of $T^{1,0} X$ and $T^{* 0,1} X \subset \mathbb{C} T^{*} X$ be the dual bundle of $T^{0,1} X$. For $p, q \in \mathbb{N}_{0}$, the bundle of $(p, q)$ forms is denoted by $T^{* p, q} X:=\left(\Lambda^{p} T^{* 1,0} X\right) \wedge\left(\Lambda^{q} T^{* 0,1} X\right)$ and let $T^{* \bullet, \bullet} X:=\oplus_{p, q \in \mathbb{N}_{0}} T^{* p, q} X$. The induced Hermitian inner product on $T^{\bullet, \bullet} X$ and $T^{* \bullet \bullet} X$ are still denoted by $\langle\cdot \mid \cdot\rangle$. The Hermitian norms are still denoted by $|\cdot|$. Let $\Omega^{p, q}(X):=\mathscr{C}^{\infty}\left(X, T^{* p, q} X\right)$ be the space of smooth $(p, q)$-forms on $X$ and $\Omega^{\bullet \bullet \bullet}(X):=\bigoplus_{p, q \in \mathbb{N}_{0}} \Omega^{p, q}(X)$. Let $\mathscr{C}^{\infty}(X):=\Omega^{0,0}(X)$. Let $\Omega_{c}^{p, q}(X)$ be the space of smooth $(p, q)$-forms on $X$ with compact support in $X$.

Since $X$ is orientable, there exists a nowhere vanishing $(2 n+1)$-form $d v_{X}$ on $X$ such that $\left|d v_{X}\right|=1$. Let $Y \subset X$ be an open subset. We denote by $T^{1,0} Y:=\left.T^{1,0} X\right|_{Y}$ and $T^{0,1} Y:=\left.T^{0,1} X\right|_{Y}$ the induced bundles over $Y$. Suppose $\left\{\bar{L}_{j}\right\}_{j=1}^{n}$ is an orthonormal frame of $T^{1,0} Y$ with the dual (orthonormal) frame $\left\{\bar{e}_{j}\right\}_{j=1}^{n}$ of $T^{* 1,0} Y$ and $\left\{L_{j}\right\}_{j=1}^{n}$ is an orthonormal frame of $T^{0,1} Y$ with the dual (orthonormal) frame $\left\{e_{j}\right\}_{j=1}^{n}$ of $T^{* 0,1} Y$. We note that the real $2 n$-form $i^{n} \bar{e}_{1} \wedge e_{1} \wedge \ldots \wedge \bar{e}_{n} \wedge e_{n}$ on $Y$ is independent of the choice of orthonormal frame $\left\{\bar{e}_{j}\right\}_{j=1}^{n}$ and hence it is globally defined. There exists a real 1 -form $\omega_{0} \in \mathscr{C}^{\infty}\left(X, T^{*} X\right)$ such that $\left|\omega_{0}\right|=1, \omega_{0}$ is orthogonal to $T^{* 1,0} X \oplus T^{* 1,0} X$, and

$$
d v_{X}=\left(i^{n} \bar{e}_{1} \wedge e_{1} \wedge \ldots \wedge \bar{e}_{n} \wedge e_{n}\right) \wedge \omega_{0}
$$

There exists a real vector field $T \in \mathscr{C}^{\infty}(X, T X)$ such that $|T|=1$ and

$$
\left\langle T, \omega_{0}\right\rangle=1, \quad\left\langle T, e_{j}\right\rangle=\left\langle T, \bar{e}_{j}\right\rangle=0, \quad j=1, \ldots, n .
$$

Thus, $\omega_{0}$ and $T$ are uniquely determined. $\omega_{0}$ is called the uniquely determined global real 1-form. $T$ is called the uniquely determined global real vector field. We have the orthogonal decompositions with respect to the Hermitian metric $\Theta_{X}$ :

$$
\begin{aligned}
\mathbb{C} T X & =T^{1,0} X \oplus T^{0,1} X \oplus \mathbb{C}\{T\}, \\
\mathbb{C} T^{*} X & =T^{* 1,0} X \oplus T^{* 0,1} X \oplus \mathbb{C}\left\{\omega_{0}\right\} .
\end{aligned}
$$


Definition 2.7. For $x \in X$, the Levi form on $T_{x}^{1,0} X$ is given by, for $u, v \in$ $T_{x}^{1,0} X$, we have

$$
\mathscr{L}_{x}(u, \bar{v}):=\frac{-1}{2 i}\left\langle[U, \bar{V}](x), \omega_{0}(x)\right\rangle,
$$

where $U, V \in \mathscr{C}^{\infty}\left(X, T^{1,0} X\right)$ with $U(x)=u, V(x)=v$.

Note that $\mathscr{L}_{x}$ is independent of the choice of $U$ and $V$, and for $x \in X$,

$$
\mathscr{L}_{x}(u, \bar{v})=\frac{1}{2 i}\left\langle U(x) \wedge \bar{V}(x), d \omega_{0}(x)\right\rangle .
$$

Definition 2.8. Let $\pi^{p, q}: \Lambda^{p+q} \mathbb{C} T^{*} X \longrightarrow T^{* p, q} X$ be the natural projection for $p, q \in \mathbb{N}_{0}, p+q \geq 1$. The tangential (resp. anti-tangential) CauchyRiemann operator is given by

$$
\begin{aligned}
\bar{\partial}_{b} & :=\pi^{p, q+1} \circ d: \Omega^{p, q}(X) \longrightarrow \Omega^{p, q+1}(X), \\
\partial_{b} & :=\pi^{p+1, q} \circ d: \Omega^{p, q}(X) \longrightarrow \Omega^{p+1, q}(X) .
\end{aligned}
$$

A function $f \in \mathscr{C}^{\infty}(X)$ is called CR if $\bar{\partial}_{b} f=0$. Let $(\cdot \mid \cdot)$ be the $L^{2}$ inner product on $\Omega_{c}^{\bullet \cdot \bullet}(X)$ induced by $\langle\cdot \mid \cdot\rangle$. Note that

$$
(u \mid v):=\int_{X}\langle u(x) \mid v(x)\rangle d v_{X}(x), \quad u, v \in \Omega_{c}^{\bullet \bullet \bullet}(X),
$$

where $d v_{X}=\left(\Theta_{X}^{n} / n !\right) \wedge \omega_{0}$. Let $L_{p, q}^{2}(X)$ be the completion of $\Omega_{c}^{p, q}(X)$ with respect to $(\cdot \mid \cdot)$. Let $L_{\bullet, \bullet}^{2}(X):=\bigoplus_{p, q \in \mathbb{N}_{0}} L_{p, q}^{2}(X)$. We write $L^{2}(X):=$ $L_{0,0}^{2}(X)$. We denote by $\|u\|^{2}:=(u \mid u)$ the $L^{2}$-norm on $X$. Let $\bar{\partial}_{b}^{*}$ and $\partial_{b}^{*}$ be the formal adjoints of $\bar{\partial}_{b}$ and $\partial_{b}$ with respect to $(\cdot \mid \cdot)$ respectively. We denote the Kohn Laplacian and the anti-Kohn Laplacian on $\Omega^{\bullet \bullet \bullet}(X)$ respectively by

$$
\square_{b}:=\bar{\partial}_{b} \bar{\partial}_{b}^{*}+\bar{\partial}_{b}^{*} \bar{\partial}_{b}, \quad \bar{\square}_{b}:=\partial_{b} \partial_{b}^{*}+\partial_{b}^{*} \partial_{b}
$$

\section{2. $\mathbb{R}$-action}

Let $\left(X, T^{1,0} X\right)$ be a CR manifold of $\operatorname{dim} X=2 n+1$. Let $r: X \rightarrow X$, $r(x)=r \circ x$ for $r \in \mathbb{R}$, be a group action on $X$ (see [5]). Let $\widehat{T}$ be the global 
real vector field on $X$ given by

$$
(\widehat{T} u)(x):=\left.\frac{\partial}{\partial r}(u(r \circ x))\right|_{r=0}, \quad u \in \mathscr{C}^{\infty}(X) .
$$

Definition 2.9. The $\mathbb{R}$-action is called $\mathrm{CR}$ if $\left[\widehat{T}, \mathscr{C}^{\infty}\left(X, T^{1,0} X\right)\right] \subset \mathscr{C}^{\infty}(X$, $\left.T^{1,0} X\right)$. The $\mathbb{R}$-action is called transversal, if $\mathbb{C} T_{x} X=T_{x}^{1,0} X \oplus T_{x}^{0,1} X \oplus$ $\mathbb{C} \widehat{T}(x)$ at every $x \in X$.

From now on, assume that $X$ admits a transversal and CR $\mathbb{R}$-action. We take the Hermitian metric $\langle\cdot \mid \cdot\rangle$ so that $\widehat{T}=T$. We will use the local coordinates of Baouendi-Rothschild-Trevers (BRT charts) [7, theorem 6.5] as follows.

Theorem 2.10 (BRT charts). For each point $x \in X$, there exists a coordinate neighbourhood $D=U \times I$ with coordinates $x=\left(x_{1}, \ldots, x_{2 n+1}\right)$ centered at 0 , where $U=\left\{z=\left(z_{1}, \ldots, z_{n}\right) \in \mathbb{C}^{n}:|z|<\epsilon\right\}$ and $I=\left\{x_{2 n+1} \in \mathbb{R}\right.$ : $\left.\left|x_{2 n+1}\right|<\epsilon_{0}\right\}, \epsilon, \epsilon_{0}>0, z=\left(z_{1}, \ldots, z_{n}\right)$ and $z_{j}=x_{2 j-1}+\sqrt{-1} x_{2 j}, j=$ $1, \ldots, n$, such that

$$
T=\frac{\partial}{\partial x_{2 n+1}} \quad \text { on } \quad D
$$

and there exists $\phi \in \mathscr{C}^{\infty}(U, \mathbb{R})$ independent of $x_{2 n+1}$ satisfying that

$$
\left\{Z_{j}:=\frac{\partial}{\partial z_{j}}+i \frac{\partial \phi}{\partial z_{j}}(z) \frac{\partial}{\partial x_{2 n+1}}\right\}_{j=1}^{n}
$$

is a frame of $T^{1,0} D$, and $\left\{d z_{j}\right\}_{j=1}^{n} \subset T^{* 1,0} D$ is the dual frame.

Let $D=U \times I$ be a BRT chart. Let $\Omega^{p, q}(D)$ be the space of smooth $(p, q)$ forms on $D$. Let $f \in \mathscr{C}^{\infty}(D)$ and $u \in \Omega^{p, q}(D)$ with $u=\sum_{I, J} u_{I J} d z_{I} \wedge d \bar{z}_{J}$ with ordered sets $I, J$ and $u_{I J} \in \mathscr{C}^{\infty}(D)$, for all $I, J$. We have

$$
\begin{aligned}
& d f=\sum_{j=1}^{n} Z_{j}(f) d z_{j}+\sum_{j=1}^{n} \bar{Z}_{j}(f) d \bar{z}_{j}+T(f) \omega_{0}, \\
& \partial_{b} f=\sum_{j=1}^{n} Z_{j}(f) d z_{j}, \quad \bar{\partial}_{b} f=\sum_{j=1}^{n} \bar{Z}_{j}(f) d \bar{z}_{j}, \\
& \partial_{b} u=\sum_{I, J}\left(\partial_{b} u_{I J}\right) \wedge d z_{I} \wedge d \bar{z}_{J}, \quad \bar{\partial}_{b} u=\sum_{I, J}\left(\bar{\partial}_{b} u_{I J}\right) \wedge d z_{I} \wedge d \bar{z}_{J} .
\end{aligned}
$$


For $u \in \Omega^{p, q}(X)$, let $\mathcal{L}_{T} u$ be the Lie derivative of $u$ in the direction of $T$. For simplicity, we write $T u$ to denote $\mathcal{L}_{T} u$. Since the $\mathbb{R}$-action is CR, $T u \in$ $\Omega^{p, q}(X)$. On a BRT chart $D$, for $u \in \Omega^{p, q}(D)$ with $u=\sum_{I, J} u_{I J} d z_{I} \wedge d \bar{z}_{J}$, we have $T u=\sum_{I, J}\left(T u_{I J}\right) \wedge d z_{I} \wedge d \bar{z}_{J}$ on $D$.

The Levi form $\mathscr{L}$ in a BRT chart $D \subset X$ has the form

$$
\mathscr{L}=\left.\partial \bar{\partial} \phi\right|_{T^{1,0} X}
$$

Indeed, the global real 1 -form is $\omega_{0}=d x_{2 n+1}-\sum_{j=1}^{n}\left(i \frac{\partial \phi}{\partial z_{j}} d z_{j}-i \frac{\partial \phi}{\partial \bar{z}_{j}} d \bar{z}_{j}\right)$ on $D$.

From now on, we assume that $\Theta_{X}$ is $\mathbb{R}$-invariant. Let $D=U \times I$ be a BRT chart. The real $(1,1)$ form $\Theta=\Theta_{U}$ on $U$ is defined by, for $x=\left(z, x_{2 n+1}\right) \in D$,

$$
\Theta(z):=\Theta_{X}(x)
$$

Note that it is independent of $x_{2 n+1}$. More precisely,

$$
\Theta(z)=\sqrt{-1} \sum_{j, k=1}^{n}\left\langle Z_{j} \mid Z_{k}\right\rangle(x) d z_{j} \wedge d \bar{z}_{k} .
$$

Note that for another BRT coordinates $D=\widetilde{U} \times \widetilde{I}, y=\left(w, y_{2 n+1}\right)$, there exist biholomorphic map $H \in \mathscr{C}^{\infty}(U, \tilde{U})$ and $G \in \mathscr{C}^{\infty}(U, \mathbb{R})$ such that $H(z)=w$, for all $z \in U, y_{2 n+1}=x_{2 n+1}+G(z)$, for all $\left(z, x_{2 n+1}\right) \in U \times I$ and $\tilde{U}=H(U)$, $\tilde{I}=I+G(U)$. We deduce that $\Theta$ is independent of the choice of BRT coordinates, i.e., $\Theta=\Theta_{U}=\Theta_{\widetilde{U}}$.

Until further notice, we work on a BRT chart $D=U \times I$. For $p, q \in \mathbb{N}_{0}$, let $T^{* p, q} U$ be the bundle of $(p, q)$ forms on $U$ and let $T^{* \bullet, \bullet} U:=\oplus_{p, q \in \mathbb{N}_{0}} T^{* p, q} U$. For $p, q \in \mathbb{N}_{0}$, let $T^{p, q} U$ be the bundle of $(p, q)$ vector fields on $U$ and let $T^{\bullet \bullet \bullet} U:=\oplus_{p, q \in \mathbb{N}_{0}} T^{p, q} U$. The $(1,1)$ form $\Theta$ induces Hermitian metrics on $T^{\bullet \bullet \bullet} U$ and $T^{* \bullet \bullet \bullet} U$. We shall use $\langle\cdot, \cdot\rangle_{h}$ to denote all the induced Hermitian metrics. The volume form on $U$ induced by $\Theta$ is given by $d \lambda(z):=\Theta^{n} / n$ !. Thus, the volume form $d v_{X}$ can be represented by

$$
d v_{X}(x)=d \lambda(z) \wedge d x_{2 n+1} \text { on } D .
$$


The $L^{2}$-inner product on $\Omega_{c}^{\bullet \bullet \bullet}(U)$ with respect to $\Theta$ is given by

$$
\left\langle s_{1}, s_{2}\right\rangle_{L^{2}(U)}:=\int_{U}\left\langle s_{1}(z), s_{2}(z)\right\rangle_{h} d \lambda(z), \quad s_{1}, s_{2} \in \Omega_{c}^{\bullet, \bullet}(U) .
$$

Let $t \in \mathbb{R}$ be fixed. The $L^{2}$-inner product on $\Omega_{c}^{\bullet, \bullet}(U)$ with respect to $\Theta$ and $e^{-2 t \phi(z)}$ is given by

$$
\left\langle s_{1}, s_{2}\right\rangle_{L^{2}\left(U, e^{-2 t \phi}\right)}:=\int_{U}\left\langle s_{1}(z), s_{2}(z)\right\rangle_{h} e^{-2 t \phi(z)} d \lambda(z), s_{1}, s_{2} \in \Omega_{c}^{\bullet \bullet \bullet}(U) .
$$

Let $\left\{L_{j}\right\}_{j=1}^{n}$ be an orthonormal frame of $T^{0,1} D$ with the dual (orthonormal) frame $\left\{e_{j}\right\}_{j=1}^{n}$. Then $\left\{\bar{L}_{j}\right\}_{j=1}^{n}$ is an orthonormal frame of $T^{1,0} D$ with the dual (orthonormal) frame $\left\{\bar{e}_{j}\right\}_{j=1}^{n}$. Since $\Theta_{X}$ is $\mathbb{R}$-invariant, there exist $c_{j}^{k}=c_{j}^{k}(z), w_{j}^{k}=w_{j}^{k}(z) \in \mathscr{C}^{\infty}(U), j, k=1, \ldots, n$, satisfying $\sum_{k=1}^{n} c_{j}^{k} w_{k}^{l}=$ $\delta_{j}^{l}$, for all $j, l=1, \ldots, n$, such that for $j=1, \ldots, n$,

$$
\bar{L}_{j}=\sum_{k=1}^{n} \bar{c}_{j}^{k} Z_{k}, \bar{e}_{j}=\sum_{k=1}^{n} \bar{w}_{k}^{j} d z_{k}, \quad L_{j}=\sum_{k=1}^{n} c_{j}^{k} \bar{Z}_{k}, \quad e_{j}=\sum_{k=1}^{n} w_{k}^{j} d \bar{z}_{k} .
$$

We can check that $\left\{w_{j}:=\sum_{k=1}^{n} \bar{c}_{j}^{k} \frac{\partial}{\partial z_{k}}\right\}_{j=1}^{n}$ and $\left\{\bar{w}_{j}:=\sum_{k=1}^{n} c_{j}^{k} \frac{\partial}{\partial \bar{z}_{k}}\right\}_{j=1}^{n}$ are orthonormal frames for $T^{1,0} U$ and $T^{0,1} U$ with respect to $\Theta$ respectively, and $\left\{\bar{e}_{j}\right\}_{j=1}^{n}$ and $\left\{e_{j}\right\}_{j=1}^{n}$ are dual frames for $\left\{w_{j}\right\}_{j=1}^{n}$ and $\left\{\bar{w}_{j}\right\}_{j=1}^{n}$ respectively. We also write $w^{j}$ and $\bar{w}^{j}$ to denote $\bar{e}_{j}$ and $e_{j}$ respectively, $j=1, \ldots, n$.

\section{3. $\mathbb{R}$-equivariant $\mathrm{CR}$ line bundle}

Let $L$ be a complex line bundle over a CR manifold $X$. For $p, q \in \mathbb{N}_{0}$, let $\Omega^{p, q}(X, L)$ be the space of smooth $(p, q)$ forms of $X$ with values in $L$ and let $\Omega^{\bullet \bullet \bullet}(X, L):=\oplus_{p, q \in \mathbb{N}_{0}} \Omega^{p, q}(X, L)$. Let $\Omega_{c}^{p, q}(X, L)$ be the subspace of $\Omega^{p, q}(X, L)$ whose elements have compact support in $X$ and let $\Omega_{c}^{\bullet, \bullet}(X, L):=$ $\oplus_{p, q \in \mathbb{N}_{0}} \Omega_{c}^{p, q}(X, L)$. Let $h^{L}$ be a Hermitian fiber metric on $L$. We denote by $\Psi$ the local weights of the Hermitian metric: if $e_{L}$ is a local trivializing section of $L$ on an open subset $Y \subset X$, then the local weight of $h^{L}$ with respect to $e_{L}$ is the function $\Phi \in \mathscr{C}^{\infty}(Y, \mathbb{R})$ for $\left|e_{L}\right|_{h^{L}}^{2}=e^{-2 \Phi}$ on $Y$.

Let $X$ be a $C R$ manifold with a transversal CR $\mathbb{R}$-action. We say that a complex line bundle $L$ is $\mathbb{R}$-equivariant and CR, if there exists an open cover $X=\cup_{j} D_{j}$ and the associated local trivialization frame $\left\{s_{j}\right\}$ such that the 
transition functions are $\mathrm{CR}$ and annihilated by $T$, where $T$ is induced by a transversal CR $\mathbb{R}$-action on $X$. In this case, we say $\left\{s_{j}\right\}$ a $\mathbb{R}$-equivariant $\mathrm{CR}$ frame of $L$ with respect to the cover $X=\cup_{j} D_{j}$. Furthermore, we can define

$$
T: \Omega^{\bullet \bullet \bullet}(X, L) \rightarrow \Omega^{\bullet \bullet \bullet}(X, L), \quad T s:=\left(T u_{j}\right) \otimes s_{j}
$$

with $u_{j} \in \Omega^{\bullet \bullet \bullet}\left(D_{j}\right)$. Since $\left\{s_{j}\right\}$ is a $\mathbb{R}$-equivariant CR frame, $T$ is welldefined.

For a $\mathbb{R}$-equivariant and CR line bundle $L$ over a CR manifold $X$ with a transversal CR $\mathbb{R}$-action, we say a Hermitian metric $h^{L}$ on $L$ is $\mathbb{R}$-invariant, if for every element $e_{L}$ in the $\mathbb{R}$-equivariant $\mathrm{CR}$ frame of $L$ with $\left|e_{L}\right|_{h^{L}}^{2}=e^{-2 \Phi}$ we have $T \Phi=0$.

These notions can be extended to the $\mathbb{R}$-equivariant $\mathrm{CR}$ vector bundles $E$ with $\mathbb{R}$-invariant Hermtian metric $h^{E}$, refer to [8, 5]. For example, $T^{1,0} X$ and $h^{T^{1,0}}$ over a CR manifold $X$ with a transversal CR $\mathbb{R}$-action are fulfilled, and in this case, we say the Hermitian metric $\Theta_{X}$ is $\mathbb{R}$-invariant.

For a CR manifold $X$ with a transversal CR $\mathbb{R}$-action endowed with an $\mathbb{R}$-invariant Hermitian metric $\Theta_{X}$ on $X$, let $K_{X}^{*}:=\operatorname{det}\left(T^{1,0} X\right)$ be the determinant of $T^{1,0} X$ and let $h^{K_{X}^{*}}$ be Hermitian metric on $K_{X}^{*}$ induced by $\Theta_{X}$. Then, $K_{X}^{*}$ is a $\mathbb{R}$-equivariant CR line bundle over $X$ and $h^{K_{X}^{*}}$ is a $\mathbb{R}$-invariant Hermitian metric on $K_{X}^{*}$.

\section{CR Bochner-Kodaira-Nakano Formula}

We firstly provide generalized Kähler identities on CR manifolds with transversal CR $\mathbb{R}$-action by the Fourier transform. Consequently we give a proof of Bochner-Kodaira-Nakano formula in this setting, which is standard in the case of complex manifolds. Moreover we show Bochner-KodairaNakano formula for $\mathbb{R}$-equivariant CR line bundle. In this section, we assume that $X$ admits a transversal CR $\mathbb{R}$-action.

\subsection{The Fourier transform}

Let $D=U \times I$ be a BRT chart. Let $\Omega_{c}^{p, q}(D)$ be the space of smooth $(p, q)$ forms on $D$ with compact support in $D$. Let $\Omega_{c}^{\bullet \bullet \bullet}(D):=\bigoplus_{p, q \in \mathbb{N}_{0}} \Omega_{c}^{p, q}(D)$. 
We write $\mathscr{C}_{c}^{\infty}(D):=\Omega_{c}^{0,0}(D)$. Let $f \in \mathscr{C}_{c}^{\infty}(D)$. We write

$$
f=f(x)=f\left(z, x_{2 n+1}\right) .
$$

For each fixed $x_{2 n+1} \in I, f\left(\cdot, x_{2 n+1}\right) \in \mathscr{C}_{c}^{\infty}(U)$. For each fixed $z \in U$, $f(z, \cdot) \in \mathscr{C}_{c}^{\infty}(I)$. Let $p, q \in \mathbb{N}_{0}, u \in \Omega_{c}^{p, q}(D)$. We write

$$
u=\sum_{I, J} u_{I J} d z_{I} \wedge d \bar{z}_{J} \in \Omega_{c}^{p, q}(D)
$$

and we always assume that the summation is performed only over increasingly ordered indices $I=i_{1}<i_{2}<\ldots<i_{p}, J=j_{1}<j_{2}<\ldots<j_{q}$, and $u_{I J} \in \mathscr{C}_{c}^{\infty}(D)$, for all $I, J$. For each fixed $z \in U, u_{I J}(z, \cdot) \in \mathscr{C}_{c}^{\infty}(I)$.

Definition 3.1. The Fourier transform of the function $f \in \mathscr{C}_{c}^{\infty}(D)$ with respect to $x_{2 n+1}$, denoted by $\widehat{f}$, is defined by

$$
\widehat{f}(z, t):=\int_{-\infty}^{\infty} e^{-i t x_{2 n+1}} f\left(z, x_{2 n+1}\right) d x_{2 n+1} \in \mathscr{C}^{\infty}(U \times \mathbb{R}) .
$$

The Fourier transform of the form $u=\sum_{I, J} u_{I J} d z_{I} \wedge d \bar{z}_{J} \in \Omega_{c}^{p, q}(D)$ with respect to $x_{2 n+1}$, denoted by $\widehat{u}$, is defined by

$$
\widehat{u}(z, t)=\sum_{I, J} \widehat{u}_{I J}(z, t) d z_{I} \wedge d \bar{z}_{J} \in \Omega^{p, q}(U \times \mathbb{R}):=\mathscr{C}^{\infty}\left(U \times \mathbb{R}, T^{* p, q} U\right) .
$$

Note that $\widehat{f} \in \mathscr{C}^{\infty}(U \times \mathbb{R})$ and $\widehat{f}(\cdot, t) \in \mathscr{C}_{c}^{\infty}(U)$ for every $t \in \mathbb{R}$. Similarly, $\widehat{u} \in \Omega^{p, q}(U \times \mathbb{R})$ and $\widehat{u}(\cdot, t) \in \Omega_{c}^{p, q}(U)$ for every $t \in \mathbb{R}$. From Parserval formula, we have for $u, v \in \Omega_{c}^{p, q}(D)$,

$$
\int_{-\infty}^{\infty}\left\langle u\left(z, x_{2 n+1}\right) \mid v\left(z, x_{2 n+1}\right)\right\rangle d x_{2 n+1}=(1 / 2 \pi) \int_{-\infty}^{\infty}\langle\widehat{u}(z, t), \widehat{v}(z, t)\rangle_{h} d t,
$$

for every $z \in U$. By using integration by parts, we have for $u \in \Omega_{c}^{p, q}(D)$,

$$
-\sqrt{-1} \widehat{T u}=t \widehat{u}, \quad \text { i.e., } \quad-\sqrt{-1} \frac{\widehat{\partial u}}{\partial x_{2 n+1}}(z, t)=t \widehat{u}(z, t) .
$$

Let $t \in \mathbb{R}$ be fixed. Let $|(z, 1)|_{h}^{2}:=e^{-2 t \phi(z)}$ be the Hermitian metric on the trivial line bundle $U \times \mathbb{C}$ over $U$. The Chern connection of $\left(U \times \mathbb{C}, e^{-2 t \phi}\right)$ 
is given by

$$
\nabla^{\left(U \times \mathbb{C}, e^{-2 t \phi}\right)}=\nabla^{1,0}+\nabla^{0,1}, \quad \nabla^{1,0}=\partial-2 t \partial \phi, \quad \nabla^{0,1}=\bar{\partial} .
$$

Indeed, the Chern connection is given by $\nabla^{\left(U \times \mathbb{C}, e^{-2 t \phi}\right)}=d+h^{-1} \partial h=d+$ $e^{2 t \phi} \partial\left(e^{-2 t \phi}\right)$. Thus, the curvature of $\left(U \times \mathbb{C}, e^{-2 t \phi}\right)$ is

$$
R^{\left(U \times \mathbb{C}, e^{-2 t \phi}\right)}=\left(\nabla^{\left(U \times \mathbb{C}, e^{-2 t \phi}\right)}\right)^{2}=2 t \partial \bar{\partial} \phi .
$$

We can identify $\partial \bar{\partial} \phi$ with Levi form $\mathscr{L}$ and write

$$
R^{\left(U \times \mathbb{C}, e^{-2 t \phi}\right)}=2 t \mathscr{L}
$$

Moreover, we will identify $\Omega^{\bullet \bullet \bullet}(U)$ and $\Omega_{c}^{\bullet \bullet \bullet}(U)$ with $\Omega^{\bullet \bullet \bullet}(U, U \times \mathbb{C})$ and $\Omega_{c}^{\bullet, \bullet}(U, U \times \mathbb{C})$ respectively. The following result means that locally the Fourier transform commutes with tangential Cauchy-Riemann operator with respect to certain weight function. For the convenience of reader, here we give the proof obtained in [9].

Proposition $3.2([9])$. With the notations used above, for $u \in \Omega_{c}^{\bullet \bullet \bullet}(D)$, we have

$$
\begin{aligned}
& \widehat{\bar{\partial}_{b} u}=e^{-t \phi} \bar{\partial}\left(e^{t \phi} \widehat{u}\right) \quad \text { on } U \times \mathbb{R}, \\
& \widehat{\bar{\partial}_{b}^{*} u}=e^{-t \phi} \bar{\partial}^{*}\left(e^{t \phi} \widehat{u}\right) \quad \text { on } U \times \mathbb{R}, \\
& \widehat{\partial_{b} u}=e^{-t \phi} \nabla^{1,0}\left(e^{t \phi} \widehat{u}\right) \quad \text { on } U \times \mathbb{R}, \\
& \widehat{\partial_{b}^{*} u}=e^{-t \phi} \nabla^{1,0 *}\left(e^{t \phi} \widehat{u}\right) \quad \text { on } U \times \mathbb{R},
\end{aligned}
$$

where $\bar{\partial}^{*}, \nabla^{1,0 *}$ are the formal adjoints of $\bar{\partial}, \nabla^{1,0}$ with respect to $\langle\cdot, \cdot\rangle_{L^{2}\left(U, e^{-2 t \phi}\right)}$ respectively and $\bar{\partial}_{b}^{*}, \partial_{b}^{*}$ are the formal adjoints of $\bar{\partial}_{b}, \partial_{b}$ with respect to $(\cdot \mid \cdot)$ respectively.

Proof. Let $u=\sum_{I, J} u_{I J} d z_{I} \wedge d \bar{z}_{J}$. By $\bar{\partial}_{b} u=\sum_{I, J} \sum_{j=1}^{n}\left(\frac{\partial u_{I J}}{\partial \bar{z}_{j}}-i \frac{\partial \phi}{\partial \bar{z}_{j}} \frac{\partial u_{I J}}{\partial x_{2 n+1}}\right) d \bar{z}_{j} \wedge$ $d z_{I} \wedge d \bar{z}_{J}$

$$
\left(\widehat{\bar{\partial}_{b} u}\right)(z, t)=\sum_{I, J} \sum_{j=1}^{n}\left(\frac{\partial \widehat{u}_{I J}}{\partial \bar{z}_{j}}(z, t)+t \frac{\partial \phi}{\partial \bar{z}_{j}}(z) \widehat{u}_{I J}(z, t)\right) d \bar{z}_{j} \wedge d z_{I} \wedge d \bar{z}_{J}
$$




$$
\begin{aligned}
& =e^{-t \phi(z)} \bar{\partial}\left(e^{t \phi} \sum_{I, J} \widehat{u}_{I J} d z_{I} \wedge d \bar{z}_{J}\right)(z, t) \\
& =\left(e^{-t \phi} \bar{\partial}\left(e^{t \phi} \widehat{u}\right)\right)(z, t) .
\end{aligned}
$$

Thus the first equality holds. From Parserval's formula, for $u, v \in \Omega_{c}^{\bullet, \bullet}(D)$,

$$
\begin{aligned}
\left(\bar{\partial}_{b} u \mid v\right) & =\int_{D}\left\langle\bar{\partial}_{b} u \mid v\right\rangle d \lambda(z) d x_{2 n+1} \\
& \left.=\int_{U}\left((2 \pi)^{-1} \int_{-\infty}^{\infty} \widehat{\left\langle\bar{\partial}_{b} u\right.}(z, t), \widehat{v}(z, t)\right\rangle_{h} d t\right) d \lambda(z) \\
& =(2 \pi)^{-1} \int_{-\infty}^{\infty} \int_{U}\left\langle e^{-t \phi} \bar{\partial}\left(e^{t \phi} \widehat{u}\right), \widehat{v}\right\rangle_{h} d \lambda(z) d t \\
& =(2 \pi)^{-1} \int_{-\infty}^{\infty}\left\langle\bar{\partial}\left(e^{t \phi} \widehat{u}\right), e^{t \phi} \widehat{v}\right\rangle_{L^{2}\left(U, e^{-2 t \phi}\right)} d t \\
& =(2 \pi)^{-1} \int_{-\infty}^{\infty}\left\langle e^{t \phi} \widehat{u}, \bar{\partial}^{*}\left(e^{t \phi} \widehat{v}\right)\right\rangle_{L^{2}\left(U, e^{-2 t \phi}\right)} d t \\
& =(2 \pi)^{-1} \int_{-\infty}^{\infty} \int_{U}\left\langle\widehat{u}, e^{-t \phi} \bar{\partial}^{*}\left(e^{t \phi} \widehat{v}\right)\right\rangle_{h} d \lambda(z) d t .
\end{aligned}
$$

Meanwhile, we have

$$
\left(\bar{\partial}_{b} u \mid v\right)=\left(u \mid \bar{\partial}_{b}^{*} v\right)=(2 \pi)^{-1} \int_{-\infty}^{\infty} \int_{U}\left\langle\widehat{u}, \widehat{\bar{\partial}_{b}^{*} v}\right\rangle_{h} d \lambda(z) d t
$$

Thus the second equality holds. The proofs of the rest equalities are simi -lar.

\subsection{Generalized Kähler identities and CR Bochner type formula}

Analogue to [13, (1.4.32)], we define the Lefschetz operator $\Theta_{X} \wedge \cdot$ on $\Lambda^{\bullet \bullet \bullet}\left(T^{*} X\right)$ and its adjoint $\Lambda=i\left(\Theta_{X}\right)$ with respect to the Hermitian inner product $\langle\cdot \mid \cdot\rangle$ associated with $\Theta_{X}$. The Hermitian torsion of $\Theta_{X}$ is defined by

$$
\mathcal{T}:=\left[\Lambda, \partial_{b} \Theta_{X}\right]
$$

Let $D=U \times I$ be a BRT chart and let $\left\{\bar{L}_{j}\right\}_{j=1}^{n} \subset T^{1,0} D,\left\{\bar{e}_{j}\right\}_{j=1}^{n} \subset T^{* 1,0} D$, $\left\{w_{j}\right\}_{j=1}^{n} \subset T^{1,0} U$ be as in (2.20). Let $i_{L_{j}}$ and $i_{\bar{L}_{j}}$ be the adjoints of $e_{j} \wedge$ and 
$\bar{e}_{j} \wedge$ respectively. We have that

$$
\Theta_{X} \wedge \cdot=\sqrt{-1} \bar{e}_{j} \wedge e_{j} \wedge \cdot, \quad \Lambda=-\sqrt{-1} i_{L_{j}} i_{\bar{L}_{j}} \quad \text { on } D
$$

Since $\partial \Theta(z)=\partial_{b} \Theta_{X}(x)$ on $D$, and $\Theta \wedge \cdot=\sqrt{-1} \bar{e}_{j} \wedge e_{j} \wedge \cdot, \Lambda=$ $-\sqrt{-1} i_{\bar{w}_{j}} i_{w_{j}}$ on $U$, see [13, 1.4.32], we have $\mathcal{T}=\left[\Lambda, \partial_{b} \Theta\right]=[\Lambda, \partial \Theta]$ on $\Omega^{\bullet \bullet \bullet}(D)$, which is independent of $x_{2 n+1}$. We remark that $\mathcal{T}$ is a differential operator of order zero. With respect to the Hermitian inner product $\langle\cdot \mid \cdot\rangle$ associated with $\Theta_{X}$, we have the adjoint operator $\mathcal{T}^{*}$, the conjugate operator $\overline{\mathcal{T}}$ and the adjoint of the conjugate operator $\overline{\mathcal{T}}^{*}$ for $\mathcal{T}$.

\subsubsection{The differential form case}

Proposition 3.3 (Generalized Kähler identities in the CR setting). We have on $\Omega^{\bullet \bullet \bullet}(X)$,

$$
\begin{aligned}
& {\left[\bar{\partial}_{b}^{*}, \Theta_{X} \wedge\right]=\sqrt{-1}\left(\partial_{b}+\mathcal{T}\right),} \\
& {\left[\partial_{b}^{*}, \Theta_{X} \wedge\right]=-\sqrt{-1}\left(\bar{\partial}_{b}+\overline{\mathcal{T}}\right),} \\
& {\left[\Lambda, \bar{\partial}_{b}\right]=-\sqrt{-1}\left(\partial_{b}^{*}+\mathcal{T}^{*}\right),} \\
& {\left[\Lambda, \partial_{b}\right]=\sqrt{-1}\left(\bar{\partial}_{b}^{*}+\overline{\mathcal{T}}^{*}\right) .}
\end{aligned}
$$

Proof. Since the both side of these identities are globally defined, we can check on a BRT chart. Now, we work on a BRT chart $D=U \times I$. For this purpose, we will use the generalized Kähler identity for the line bundle $\left(U \times \mathbb{C}, e^{-2 t \phi}\right)$ over $U$ as follows:

$$
\begin{aligned}
& {\left[\bar{\partial}^{*}, \Theta \wedge\right]=\sqrt{-1}\left(\nabla^{1,0}+\mathcal{T}\right),} \\
& {\left[\nabla^{1,0 *}, \Theta \wedge\right]=-\sqrt{-1}(\bar{\partial}+\overline{\mathcal{T}}),} \\
& {[\Lambda, \bar{\partial}]=-\sqrt{-1}\left(\nabla^{1,0 *}+\mathcal{T}^{*}\right),} \\
& {\left[\Lambda, \nabla^{1,0}\right]=\sqrt{-1}\left(\bar{\partial}^{*}+\overline{\mathcal{T}}^{*}\right) .}
\end{aligned}
$$

Let $u, v \in \Omega_{c}^{\bullet, \bullet}(D)$. Let $s_{1}(z):=e^{t \phi(z)} \widehat{u}(z, t) \in \Omega^{\bullet \bullet \bullet}(U \times \mathbb{R}), s_{2}(z):=$ $e^{t \phi(z)} \widehat{v}(z, t) \in \Omega^{\bullet \bullet \bullet}(U \times \mathbb{R})$. We will show that $\left(\left[\bar{\partial}_{b}^{*}, \Theta_{X} \wedge\right] u \mid v\right)=\left(\sqrt{-1}\left(\partial_{b}+\right.\right.$ $\mathcal{T}) u \mid v)$, and thus the first identity holds. In fact, by the equality $\left[\bar{\partial}^{*}, \Theta \wedge\right]=$ $\sqrt{-1}\left(\nabla^{1,0}+\mathcal{T}\right)$, we obtain that

$$
2 \pi\left(\left[\bar{\partial}_{b}^{*}, \Theta_{X} \wedge\right] u \mid v\right)=\int_{-\infty}^{+\infty}\left\langle\left[\bar{\partial}_{b}^{*}, \widehat{\Theta_{X} \wedge}\right] u \mid \widehat{v}\right\rangle_{L^{2}(U)} d t
$$




$$
\begin{aligned}
& =\int_{-\infty}^{+\infty}\left\langle\left[\bar{\partial}^{*}, \Theta \wedge\right] s_{1}, s_{2}\right\rangle_{L^{2}\left(U, e^{-2 t \phi}\right)} d t \\
& =\int_{-\infty}^{+\infty}\left\langle\sqrt{-1}\left(\nabla^{1,0}+\mathcal{T}\right) s_{1}, s_{2}\right\rangle_{L^{2}\left(U, e^{-2 t \phi}\right)} d t \\
& =\int_{-\infty}^{+\infty}\left\langle\sqrt{-1}\left(\widehat{\partial_{b}+} \mathcal{T}\right) u \mid \widehat{v}\right\rangle_{L^{2}(U)} d t \\
& =2 \pi\left(\sqrt{-1}\left(\partial_{b}+\mathcal{T}\right) u \mid v\right) .
\end{aligned}
$$

Here we use the second identity in Proposition 3.2. The proof of the rest is similar.

The generalized Kähler identities implies the CR version of the BochnerKodaira-Nakano formula with $\mathbb{R}$-action. The proof is standard, see e.g. 13, Theorem 1.4.12], however, it is still interesting to identify the term $2 \mathscr{L T}$ in the following argument.

Theorem 3.4 (CR Bochner-KodairaNakano formula for differential forms). With the notations used above, we have on $\Omega^{\bullet \bullet \bullet}(X)$,

$$
\square_{b}=\bar{\square}_{b}+[2 \mathscr{L} T, \Lambda]+\left[\partial_{b}, \mathcal{T}^{*}\right]-\left[\bar{\partial}_{b}, \overline{\mathcal{T}}^{*}\right]
$$

If $X$ is Levi flat, i.e., $\mathscr{L}=0$, then

$$
\square_{b}=\bar{\square}_{b}+\left[\partial_{b}, \mathcal{T}^{*}\right]-\left[\bar{\partial}_{b}, \overline{\mathcal{T}}^{*}\right]
$$

If $X$ is Kähler, i.e., $d \Theta_{X}=0$, then

$$
\square_{b}=\bar{\square}_{b}+[2 \mathscr{L} T, \Lambda] .
$$

If $X$ is Levi flat and Kähler, then

$$
\square_{b}=\bar{\square}_{b} .
$$

Proof. By the generalized Kähler identities above, we have $\bar{\partial}_{b}^{*}=-\sqrt{-1}\left[\Lambda, \partial_{b}\right]-\overline{\mathcal{T}}^{*}$. Thus

$$
\square_{b}=\left[\bar{\partial}_{b}, \bar{\partial}_{b}^{*}\right]=-\sqrt{-1}\left[\bar{\partial}_{b},\left[\Lambda, \partial_{b}\right]\right]-\left[\bar{\partial}_{b}, \overline{\mathcal{T}}^{*}\right] .
$$


We define

$$
R_{b}:=\left[\partial_{b}, \bar{\partial}_{b}\right]
$$

By Jacobi identity, we see that

$$
\left[\bar{\partial}_{b},\left[\Lambda, \partial_{b}\right]\right]=\left[\Lambda,\left[\partial_{b}, \bar{\partial}_{b}\right]\right]+\left[\partial_{b},\left[\bar{\partial}_{b}, \Lambda\right]\right]=\left[\Lambda, R_{b}\right]+\left[\partial_{b},\left[\bar{\partial}_{b}, \Lambda\right]\right] .
$$

The third identity in Proposition 3.3 implies

$$
\left[\partial_{b},\left[\bar{\partial}_{b}, \Lambda\right]\right]=\sqrt{-1}\left[\partial_{b}, \partial_{b}^{*}\right]+\sqrt{-1}\left[\partial_{b}, \mathcal{T}^{*}\right]=\sqrt{-1} \square_{b}+\sqrt{-1}\left[\partial_{b}, \mathcal{T}^{*}\right] .
$$

Thus we have

$$
\square_{b}=\bar{\square}_{b}+\left[\sqrt{-1} R_{b}, \Lambda\right]+\left[\partial_{b}, \mathcal{T}^{*}\right]-\left[\bar{\partial}_{b}, \overline{\mathcal{T}}^{*}\right],
$$

where

$$
\sqrt{-1} R_{b}=\sqrt{-1}\left(\partial_{b} \bar{\partial}_{b}+\bar{\partial}_{b} \partial_{b}\right)=2 \mathscr{L} \circ T
$$

by $\mathscr{L}=\partial \bar{\partial} \phi$ and $T=\frac{\partial}{\partial x_{2 n+1}}$ in the BRT charts.

\subsubsection{The line bundle case}

More generally, we consider the Bochner-Kodaira-Nakano formula with $\mathbb{R}$-action for $\mathrm{CR}$ line bundle. We assume that $L$ is a $\mathbb{R}$-equivariant $\mathrm{CR}$ line bundle over $X$ with a $\mathbb{R}$-invariant Hermitian metric $h^{L}$ on $L$. For $p, q \in \mathbb{N}_{0}$, let

$$
\bar{\partial}_{b}^{L}: \Omega^{p, q}(X, L) \rightarrow \Omega^{p, q+1}(X, L)
$$

be the tangential Cauchy-Riemann operator with values in $L$. Let $(\cdot \mid \cdot)_{L}$ be the $L^{2}$ inner product on $\Omega_{c}^{\bullet \bullet \bullet}(X, L)$ induced by $\langle\cdot \mid \cdot\rangle$ and $h^{L}$. Let

$$
\bar{\partial}_{b}^{L *}: \Omega^{p, q+1}(X, L) \rightarrow \Omega^{p, q}(X, L)
$$

be the formal adjoint of $\bar{\partial}_{b}^{L}$ with respect to $(\cdot \mid \cdot)_{L}$. Kohn Laplacian with respect to $L$ is

$$
\square_{b}^{L}:=\bar{\partial}_{b}^{L} \bar{\partial}_{b}^{L *}+\bar{\partial}_{b}^{L *} \bar{\partial}_{b}^{L}
$$


The connection on $L$ induced by $h^{L}$ is defined

$$
\nabla_{b}^{L}: \Omega^{\bullet \bullet \bullet}(X, L) \rightarrow \Omega^{\bullet \bullet \bullet}(X, L)
$$

as follows: let $e_{L}$ be a $\mathbb{R}$-equivariant $\mathrm{CR}$ frame of $L$ on a BRT chart $D \subset X$ such that

$$
\left|e_{L}\right|_{h^{L}}^{2}=e^{-2 \Phi}, \quad \Phi \in \mathscr{C}^{\infty}(D, \mathbb{R}) .
$$

Note that $\Phi$ is independent of $x_{2 n+1}$ by $T \Phi=0$. For $s \in \Omega^{\bullet \bullet \bullet}(D, L)$ with $s=u \otimes e_{L}, u \in \Omega^{\bullet \bullet \bullet}(D)$,

$$
\left(\nabla_{b}^{L}\right)^{0,1} s:=\bar{\partial}_{b}^{L} s=\bar{\partial}_{b} u \otimes e_{L}, \quad\left(\nabla_{b}^{L}\right)^{1,0} s:=\left(\partial_{b} u-2 \partial_{b} \Phi \wedge u\right) \otimes e_{L} .
$$

Then we define

$$
\nabla_{b}^{L}:=\left(\nabla_{b}^{L}\right)^{1,0}+\left(\nabla_{b}^{L}\right)^{0,1}=\left(\nabla_{b}^{L}\right)^{1,0}+\bar{\partial}_{b}^{L}
$$

Note that (3.29) is independent of the choices of $T$-invariant local CR trivializing sections and hence is globally defined. We set

$$
R_{b}^{L}:=\left(\nabla_{b}^{L}\right)^{2} .
$$

Let $\left(\nabla^{L}\right)^{1,0 *}$ is the adjoint of $\left(\nabla^{L}\right)^{1,0}$ with respect to $(\cdot \mid \cdot)_{L}$. We define the anti-Kohn Laplacian with respect to $L$ by

$$
\bar{\square}_{b}^{L}:=\left(\nabla_{b}^{L}\right)^{1,0}\left(\nabla_{b}^{L}\right)^{1,0 *}+\left(\nabla_{b}^{L}\right)^{1,0 *}\left(\nabla_{b}^{L}\right)^{1,0} .
$$

Let $R^{L} \in \Omega^{1,1}(X)$ be the curvature of $L$ induced by $h^{L}$ given by

$$
R^{L}:=2 \partial_{b} \bar{\partial}_{b} \Phi
$$

on $D$, where $\Phi$ is as in (3.27). It is easy to check that $R^{L}$ is globally defined. Let $D=U \times I$ be a BRT chart. Since $L$ is $\mathbb{R}$-equivariant on $D, L$ is a holomorphic line bundle over $U$. By the BRT charts, we can check that

$$
R_{b}^{L}=\left[\left(\nabla_{b}^{L}\right)^{1,0}, \bar{\partial}_{b}^{L}\right]=\left[\partial_{b}, \bar{\partial}_{b}\right]+2 \partial_{b} \bar{\partial}_{b} \Phi=-2 \sqrt{-1} \mathscr{L} \circ T+R^{L} .
$$

Now we can follow the proof of Theorem 3.4 to check Theorem 1.2, Firstly, by the Fourier transform as in Proposition 3.2, we have: 
Proposition 3.5. With the notations used above, let $s \in \Omega_{c}^{\bullet, \bullet}(D, L)$ with $s=u \otimes e_{L}$ and $u \in \Omega_{c}^{\bullet \bullet \bullet}(D)$. Let $\widehat{s}:=\widehat{u} \otimes e_{L}$. Then, we have

$$
\begin{aligned}
\widehat{\bar{\partial}_{b}^{L}} s & =e^{-t \phi} \bar{\partial}\left(e^{t \phi} \widehat{u}\right) \otimes e_{L} \quad \text { on } U \times \mathbb{R}, \\
\widehat{\bar{\partial}_{b}^{L *}} s & =e^{-t \phi} \bar{\partial}_{\Phi}^{*}\left(e^{t \phi} \widehat{u}\right) \otimes e_{L} \quad \text { on } U \times \mathbb{R}, \\
\widehat{\left(\nabla_{b}^{L}\right)^{1,0}} s & =e^{-t \phi} \nabla_{\Phi}^{1,0}\left(e^{t \phi} \widehat{u}\right) \otimes e_{L} \quad \text { on } U \times \mathbb{R}, \\
\left(\widehat{\left.\nabla_{b}^{L}\right)^{1,0} *} s\right. & =e^{-t \phi} \nabla_{\Phi}^{1,0 *}\left(e^{t \phi} \widehat{u}\right) \otimes e_{L} \quad \text { on } U \times \mathbb{R},
\end{aligned}
$$

where $\bar{\partial}_{\Phi}^{*}$ and $\nabla_{\Phi}^{1,0 *}:=\left(\nabla^{1,0}-2 \partial_{b} \Phi\right)^{*}$ are the formal adjoints of $\bar{\partial}$ and $\nabla_{\Phi}^{1,0}:=\nabla^{1,0}-2 \partial_{b} \Phi=\partial-2 t \partial \phi-2 \partial \Phi$ with respect to $\langle\cdot, \cdot\rangle_{L^{2}\left(U, e^{-2 t \phi-2 \Phi}\right)}$ respectively, $\bar{\partial}_{b}^{L *}$ and $\left(\nabla_{b}^{L}\right)^{1,0 *}$ are the formal adjoints of $\bar{\partial}_{b}^{L}$ and $\left(\nabla_{b}^{L}\right)^{1,0}$ with respect to $(\cdot \mid \cdot)_{L}$ respectively.

Secondly, we use Proposition 3.5 and the generalized Kähler identity for the line bundle $\left(U \times \mathbb{C}, e^{-2 t \phi-2 \Phi}\right)$ over $U$ :

$$
\begin{aligned}
{\left[\bar{\partial}_{\Phi}^{*}, \Theta \wedge\right] } & =\sqrt{-1}\left(\nabla_{\Phi}^{1,0}+\mathcal{T}\right), \\
{\left[\nabla_{\Phi}^{1,0 *}, \Theta \wedge\right] } & =-\sqrt{-1}(\bar{\partial}+\overline{\mathcal{T}}), \\
{[\Lambda, \bar{\partial}] } & =-\sqrt{-1}\left(\nabla_{\Phi}^{1,0 *}+\mathcal{T}^{*}\right), \\
{\left[\Lambda, \nabla_{\Phi}^{1,0}\right] } & =\sqrt{-1}\left(\bar{\partial}_{\Phi}^{*}+\overline{\mathcal{T}}^{*}\right),
\end{aligned}
$$

to obtain Proposition 1.1, which is analogue to Proposition 3.3 Finally, by Proposition 1.1 and (3.33), we obtain Theorem 1.2 along the argument in the proof of Theorem 3.4 .

\subsection{Versions of CR Bochner-KodairaNakano formula}

\subsubsection{The version of zero order error term}

Along the argument in [3, VII.1], we can reformulate (1.2) to eliminate the first order error term $\left[\left(\nabla_{b}^{L}\right)^{1,0}, \mathcal{T}^{*}\right]-\left[\bar{\partial}_{b}^{L}, \overline{\mathcal{T}}^{*}\right]$.

Proof of Theorem 1.3. It is clear that

$$
\bar{\square}_{b, \mathcal{T}}^{L}:=\left[\left(\nabla_{b}^{L}\right)^{1,0}+\mathcal{T},\left(\nabla_{b}^{L}\right)^{1,0 *}+\mathcal{T}^{*}\right]
$$


is a positive formally and self-adjoint, because

$$
\left(\bar{\square}_{b, \mathcal{T}}^{L} s \mid s\right)=\left\|\left(\nabla_{b}^{L}\right)^{1,0} s+\mathcal{T} s\right\|^{2}+\left\|\left(\nabla_{b}^{L}\right)^{1,0 *} s+\mathcal{T}^{*} s\right\|^{2} \geq 0
$$

for $s \in \Omega_{c}^{\bullet, \bullet}(X, L)$. We need the following two lemmas.

\section{Lemma 3.1.}

$$
\begin{aligned}
{\left[\Theta_{X} \wedge, \mathcal{T}\right] } & =3 \partial_{b} \Theta_{X} \\
{[\Lambda, \mathcal{T}] } & =-2 \sqrt{-1 \mathcal{T}^{*}}
\end{aligned}
$$

In fact, by $\left[\Theta_{X} \wedge, \partial_{b} \Theta_{X}\right]=\Theta_{X} \wedge \partial_{b} \Theta_{X} \wedge-\partial_{b} \Theta_{X} \wedge \Theta_{X} \wedge=0$, the Jacobi identity implies

$$
\begin{aligned}
{\left[\Theta_{X} \wedge, \mathcal{T}\right] } & =\left[\Theta_{X} \wedge,\left[\Lambda, \partial_{b} \Theta_{X}\right]\right] \\
& =-\left[\Lambda,\left[\partial_{b} \Theta_{X}, \Theta_{X}\right]\right]-\left[\partial_{b} \Theta_{X},\left[\Theta_{X} \wedge, \Lambda\right]\right] \\
& =-\left[\partial_{b} \Theta_{X},\left[\Theta_{X} \wedge, \Lambda\right]\right] .
\end{aligned}
$$

Since $\partial_{b} \Theta_{X} \in \Omega^{2,1}(X)$ and $\left[\Theta_{X} \wedge, \Lambda\right] u=(p+q-n) u$ for $u \in \Omega^{p, q}(X)$ with $\operatorname{dim}_{\mathbb{R}} X=2 n+1$, we have

$$
\begin{aligned}
-\left[\partial_{b} \Theta_{X},\left[\Theta_{X} \wedge, \Lambda\right]\right] u & =-\partial_{b} \Theta_{X} \wedge\left[\Theta_{X} \wedge, \Lambda\right] u+\left[\Theta_{X} \wedge, \Lambda\right]\left(\partial_{b} \Theta_{X} \wedge u\right) \\
& =-(p+q-n) \partial_{b} \Theta_{X} \wedge u+(p+q+3-n) \partial_{b} \Theta_{X} \wedge u \\
& =3 \partial_{b} \Theta_{X} \wedge u
\end{aligned}
$$

Thus (3.36) holds. From Proposition 1.1, we have

$$
\begin{aligned}
{[\Lambda, \mathcal{T}] } & =\left[\Lambda,-\sqrt{-1}\left[\bar{\partial}_{b}^{L *}, \Theta_{X} \wedge\right]-\left(\nabla_{b}^{L}\right)^{1,0}\right] \\
& =-\sqrt{-1}\left[\Lambda,\left[\bar{\partial}_{b}^{L *}, \Theta_{X} \wedge\right]\right]-\sqrt{-1}\left[\bar{\partial}_{b}^{L *}+\overline{\mathcal{T}}^{*}\right] .
\end{aligned}
$$

By the Jacobi identity,

$$
\begin{aligned}
{\left[\Lambda,\left[\bar{\partial}_{b}^{L *}, \Theta_{X}\right]\right] } & =-\left[\bar{\partial}_{b}^{L *},\left[\Theta_{X} \wedge, \Lambda\right]\right]-\left[\Theta_{X},\left[\Lambda, \bar{\partial}_{b}^{L *}\right]\right] \\
& =-\bar{\partial}_{b}^{L *}\left[\Theta_{X}, \Lambda\right]+\left[\Theta_{X}, \Lambda\right] \bar{\partial}_{b}^{L *}-\left[\Theta_{X},\left[\Lambda, \bar{\partial}_{b}^{L *}\right]\right] \\
& =-\bar{\partial}_{b}^{L *}-\left[\Theta_{X},\left[\Lambda, \bar{\partial}_{b}^{L *}\right]\right] \\
& =-\bar{\partial}_{b}^{L *}-\left[\left[\bar{\partial}_{b}^{L}, \Theta_{X}\right], \Lambda\right]^{*}
\end{aligned}
$$


From $\left[\bar{\partial}_{b}^{L}, \Theta_{X}\right] s=\bar{\partial}_{b}^{L}\left(\Theta_{X} \wedge s\right)-\Theta_{X} \wedge \bar{\partial}_{b}^{L} s=\left(\bar{\partial}_{b}^{L} \Theta_{X}\right) \wedge s$, we obtain

$$
\left[\Lambda,\left[\bar{\partial}_{b}^{L *}, \Theta_{X}\right]\right]=-\bar{\partial}_{b}^{L *}-\left[\bar{\partial}_{b}^{L} \Theta_{X}, \Lambda\right]^{*}=-\bar{\partial}_{b}^{L *}+\overline{\mathcal{T}}^{*}
$$

thus $[\Lambda, \mathcal{T}]=-\sqrt{-1}\left(-\bar{\partial}_{b}^{L *}+\overline{\mathcal{T}}^{*}\right)-\sqrt{-1}\left[\bar{\partial}_{b}^{L *}+\overline{\mathcal{T}}^{*}\right]=-2 \sqrt{-1 \mathcal{T}^{*}}$, that is, (3.37) holds.

\section{Lemma 3.2.}

$$
\begin{aligned}
{\left[\left(\nabla_{b}^{L}\right)^{1,0}, \overline{\mathcal{T}}^{*}\right] } & =-\left[\left(\nabla_{b}^{L}\right)^{1,0}, \bar{\partial}_{b}^{L *}\right]=\left[\mathcal{T}, \bar{\partial}_{b}^{L *}\right] \\
-\left[\bar{\partial}_{b}^{L}, \overline{\mathcal{T}}^{*}\right] & =\left[\mathcal{T},\left(\nabla_{b}^{L}\right)^{1,0 *}+\mathcal{T}^{*}\right]+\mathcal{T}_{\Theta} .
\end{aligned}
$$

In fact, the Jacobi identity implies that

$$
-\left[\left(\nabla_{b}^{L}\right)^{1,0},\left[\Lambda,\left(\nabla_{b}^{L}\right)^{1,0}\right]\right]+\left[\Lambda,\left[\left(\nabla_{b}^{L}\right)^{1,0},\left(\nabla_{b}^{L}\right)^{1,0}\right]\right]+\left[\left(\nabla_{b}^{L}\right)^{1,0},\left[\left(\nabla_{b}^{L}\right)^{1,0}, \Lambda\right]\right]=0 .
$$

Since $\left(\nabla_{b}^{L}\right)^{1,0}\left(\nabla_{b}^{L}\right)^{1,0}=0$, we have $\left[\left(\nabla_{b}^{L}\right)^{1,0},\left[\Lambda,\left(\nabla_{b}^{L}\right)^{1,0}\right]\right]=0$. Thus we have

$$
\begin{aligned}
{\left[\left(\nabla_{b}^{L}\right)^{1,0}, \overline{\mathcal{T}}^{*}\right] } & =\left[\left(\nabla_{b}^{L}\right)^{1,0},\left(\sqrt{-1}\left[\left(\nabla_{b}^{L}\right)^{1,0 *}, \Theta_{X} \wedge\right]-\bar{\partial}_{b}^{L}\right)^{*}\right] \\
& =-\left[\left(\nabla_{b}^{L}\right)^{1,0}, \bar{\partial}_{b}^{L *}\right]-\sqrt{-1}\left[\left(\nabla_{b}^{L}\right)^{1,0},\left[\Lambda,\left(\nabla_{b}^{L}\right)^{1,0}\right]\right] \\
& =-\left[\left(\nabla_{b}^{L}\right)^{1,0}, \bar{\partial}_{b}^{L *}\right] \\
& =\left[\mathcal{T}+\sqrt{-1}\left[\bar{\partial}_{b}^{L *}, \Theta_{X}\right], \bar{\partial}_{b}^{L *}\right] \\
& =\left[\mathcal{T}, \bar{\partial}_{b}^{L *}\right]+\sqrt{-1}\left[\left[\bar{\partial}_{b}^{L *}, \Theta_{X}\right], \bar{\partial}_{b}^{L *}\right] \\
& =\left[\mathcal{T}, \bar{\partial}_{b}^{L *}\right]
\end{aligned}
$$

Thus (3.45) holds. From (3.37), $\overline{\mathcal{T}}^{*}=\frac{\sqrt{-1}}{2}[\Lambda, \mathcal{T}]$,

$$
\left[\bar{\partial}_{b}^{L}, \overline{\mathcal{T}}^{*}\right]=\frac{\sqrt{-1}}{2}\left[\bar{\partial}_{b}^{L},[\Lambda, \mathcal{T}]\right] .
$$

Jacobi identity implies that

$$
\begin{aligned}
{\left[\bar{\partial}_{b}^{L},[\Lambda, \mathcal{T}]\right] } & =\left[\Lambda,\left[\mathcal{T}, \bar{\partial}_{b}^{L}\right]\right]+\left[\mathcal{T},\left[\bar{\partial}_{b}^{L}, \Lambda\right]\right] \\
{\left[\mathcal{T}, \bar{\partial}_{b}^{L}\right] } & =\left[\bar{\partial}_{b}^{L}, \mathcal{T}\right]=\left[\bar{\partial}_{b}^{L},\left[\Lambda, \partial_{b} \Theta_{X}\right]\right] \\
& =\left[\Lambda,\left[\partial_{b} \Theta_{X}, \bar{\partial}_{b}^{L}\right]\right]+\left[\partial_{b} \Theta_{X},\left[\bar{\partial}_{b}^{L}, \Lambda\right]\right] .
\end{aligned}
$$

From $\left[\partial_{b} \Theta_{X}, \bar{\partial}_{b}^{L}\right]=\left(\partial_{b} \Theta_{X}\right) \wedge \bar{\partial}_{b}^{L}+\bar{\partial}_{b}^{L}\left(\partial_{b} \Theta_{X} \wedge\right)=\bar{\partial}_{b} \partial_{b} \Theta_{X}$ and Proposition 
1.1. we have

$$
\left[\mathcal{T}, \bar{\partial}_{b}^{L}\right]=\left[\Lambda, \bar{\partial}_{b} \partial_{b} \Theta_{X}\right]+\left[\partial_{b} \Theta_{X}, A\right],
$$

where $A:=\left[\bar{\partial}_{b}^{L}, \Lambda\right]=\sqrt{-1}\left(\left(\nabla_{b}^{L}\right)^{1,0 *}+\mathcal{T}^{*}\right)$. Thus

$$
\left[\Lambda,\left[\mathcal{T}, \bar{\partial}_{b}^{L}\right]\right]=\left[\Lambda,\left[\Lambda, \bar{\partial}_{b} \partial_{b} \Theta_{X}\right]\right]+\left[\Lambda,\left[\partial_{b} \Theta_{X}, A\right]\right] .
$$

We consider

$$
\begin{aligned}
{\left[\Lambda,\left[\partial_{b} \Theta_{X}, A\right]\right] } & =\left[A,\left[\Lambda, \partial_{b} \Theta_{X}\right]\right]-\left[\partial_{b} \Theta_{X},[A, \Lambda]\right] \\
& =[\mathcal{T}, A]+\left[\partial_{b} \Theta_{X},[\Lambda, A]\right] .
\end{aligned}
$$

By (3.36), we have $\left[\mathcal{T}, \Theta_{X} \wedge\right]=-3 \partial_{b} \Theta_{X}$. From that

$$
\left[\left(\nabla_{b}^{L}\right)^{1,0}, \Theta_{X} \wedge\right]=\left(\nabla_{b}^{L}\right)^{1,0}\left(\Theta_{X} \wedge\right)-\Theta_{X} \wedge\left(\nabla_{b}^{L}\right)^{1,0}=\partial_{b} \Theta_{X},
$$

we have

$$
\begin{aligned}
{[\Lambda, A] } & =\left[\Lambda, \sqrt{-1}\left(\left(\nabla_{b}^{L}\right)^{1,0 *}+\mathcal{T}^{*}\right)\right] \\
& =\sqrt{-1}\left[\left(\nabla_{b}^{L}\right)^{1,0}, \Theta_{X}\right]^{*}+\sqrt{-1}\left[\mathcal{T}, \Theta_{X}\right]^{*} \\
& =\sqrt{-1}\left(\partial_{b} \Theta_{X}\right)^{*}-3 \sqrt{-1}\left(\partial_{b} \Theta_{X}\right)^{*} \\
& =-2 \sqrt{-1}\left(\partial_{b} \Theta_{X}\right)^{*} .
\end{aligned}
$$

Now (3.54) becomes

$$
\left[\Lambda,\left[\partial_{b} \Theta_{X}, A\right]\right]=\left[\mathcal{T},\left[\bar{\partial}_{b}^{L}, \Lambda\right]\right]-2 \sqrt{-1}\left[\partial_{b} \Theta_{X},\left(\partial_{b} \Theta_{X}\right)^{*}\right] .
$$

From (3.49), (3.53) and (3.56) we have

$$
\begin{aligned}
{\left[\bar{\partial}_{b}^{L},[\Lambda, \mathcal{T}]\right] } & =\left[\Lambda,\left[\Lambda, \bar{\partial}_{b} \partial_{b} \Theta_{X}\right]\right]+2\left[\mathcal{T},\left[\bar{\partial}_{b}^{L}, \Lambda\right]\right]-2 \sqrt{-1}\left[\partial_{b} \Theta_{X},\left(\partial_{b} \Theta_{X}\right)^{*}\right] \\
& =2 \sqrt{-1}\left(\mathcal{T}_{\Theta}+\left[\mathcal{T},\left(\nabla_{b}^{L}\right)^{1,0 *}+\mathcal{T}^{*}\right]\right)
\end{aligned}
$$

From (3.48), we have $\left[\bar{\partial}_{b}^{L}, \overline{\mathcal{T}}^{*}\right]=-\left(\mathcal{T}_{\Theta}+\left[\mathcal{T},\left(\nabla_{b}^{L}\right)^{1,0 *}+\mathcal{T}^{*}\right]\right)$, that is, (3.46) holds.

Finally, we use (3.46) to obtain:

$$
\bar{\square}_{b}^{L}+\left[\left(\nabla_{b}^{L}\right)^{1,0}, \mathcal{T}^{*}\right]-\left[\bar{\partial}_{b}^{L}, \overline{\mathcal{T}}^{*}\right]=\bar{\square}_{b}^{L}+\left[\left(\nabla_{b}^{L}\right)^{1,0}, \mathcal{T}^{*}\right]+\mathcal{T}_{\Theta}+\left[\mathcal{T},\left(\nabla_{b}^{L}\right)^{1,0 *}+\mathcal{T}^{*}\right]
$$




$$
\begin{aligned}
& =\left[\left(\nabla_{b}^{L}\right)^{1,0}+\mathcal{T},\left(\nabla_{b}^{L}\right)^{1,0 *}+\mathcal{T}^{*}\right]+\mathcal{T}_{\Theta} \\
& =\bar{\square}_{b, \mathcal{T}}^{L}+\mathcal{T}_{\Theta} .
\end{aligned}
$$

So (1.7) follows from (1.2).

Similar to [3, VII.1] it also follows that:

Theorem 3.6. Let $\Delta_{b, \mathcal{T}}^{L}:=\left[\nabla_{b}^{L}+\mathcal{T}, \nabla_{b}^{L *}+\mathcal{T}^{*}\right]$. Then, we have on $\Omega^{\bullet}, \bullet(X, L)$,

$$
\Delta_{b, \mathcal{T}}^{L}=\square_{b}^{L}+\square_{b, \mathcal{T}}^{L}
$$

Proof. By (3.45),$\left[\mathcal{T}+\left(\nabla_{b}^{L}\right)^{1,0}, \bar{\partial}_{b}^{L *}\right]=0$. Thus, we have

$$
\begin{aligned}
\Delta_{b, \mathcal{T}}^{L} & =\left[\left(\nabla_{b}^{L}\right)^{1,0}+\bar{\partial}_{b}^{L}+\mathcal{T},\left(\nabla_{b}^{L}\right)^{1,0 *}+\bar{\partial}_{b}^{L *}+\mathcal{T}^{*}\right] \\
& =\left[\bar{\partial}_{b}^{L}, \bar{\partial}_{b}^{L *}\right]+\bar{\square}_{b, \mathcal{T}} \\
& =\square_{b}+\bar{\square}_{b, \mathcal{T}} .
\end{aligned}
$$

\subsubsection{The twisted version}

In order to establish vanishing theorems, we need another twisted version of Bochner-Kodaira-Nakano formula. The bundle $K_{X}^{*}:=\operatorname{det}\left(T^{1,0} X\right)$ is a $\mathbb{R}$ equivariant CR line bundle over $X$. The $(1,1)$ form $\Theta_{X}$ induces a $\mathbb{R}$-invariant Hermitian metric $h^{K_{X}^{*}}$ on $K_{X}^{*}$. Let $R^{K_{X}^{*}}$ be the curvature of $K_{X}^{*}$ induced by $h^{K_{X}^{*}}$.

Note that the curvature $R^{K_{X}^{*}}$ of $K_{X}^{*}$ with respect to $h^{K_{X}^{*}}$ can be represented as follows: $R^{K_{X}^{*}}=\bar{\partial}_{b} \partial_{b} \log \operatorname{det}\left(\left\langle Z_{j} \mid Z_{k}\right\rangle\right)_{j, k=1}^{n}$ on a BRT chart $D$. It is clear that $R^{K_{X}^{*}}$ is independent of the choice of BRT coordinates and hence $R^{K_{X}^{*}}$ is globally defined, i.e. $R^{K_{X}^{*}} \in \Omega^{1,1}(X)$.

Let

$$
\Psi: T^{* 0, q} X \otimes L \rightarrow T^{* n, q} X \otimes L \otimes K_{X}^{*}
$$

be the natural isometry defined as follows: Let $D=U \times I$ be a BRT chart. Let $\left\{\bar{L}_{j}\right\}_{j=1}^{n} \subset T^{1,0} D,\left\{\bar{e}_{j}\right\}_{j=1}^{n} \subset T^{* 1,0} D$ be as in (2.20). Then, for $s \in$ $T^{* 0, q} X \otimes L$,

$$
\Psi s:=\bar{e}_{1} \wedge \ldots \wedge \bar{e}_{n} \wedge s \otimes\left(\bar{L}_{1} \wedge \ldots \wedge \bar{L}_{n}\right) \in T^{* n, q} X \otimes L \otimes K_{X}^{*}
$$


It is easy to see that the definition above is independent of the choices of $\mathbb{R}$ invariant orthonormal frame $\left\{\bar{L}_{j}\right\}_{j=1}^{n} \subset T^{1,0} D$ and hence is globally defined. We have the isometry:

$$
\Psi: \Omega^{0, q}(X, L) \rightarrow \Omega^{n, q}\left(X, L \otimes K_{X}^{*}\right)
$$

Moreover, it is straightforward to see that for $s \in \Omega^{0, q}(X, L)$

$$
\bar{\partial}_{b}^{L} s=\Psi^{-1} \bar{\partial}_{b}^{L \otimes K_{X}^{*}} \Psi s, \quad \bar{\partial}_{b}^{L *} s=\Psi^{-1} \bar{\partial}_{b}^{L \otimes K_{X}^{*} *} \Psi s, \quad \square_{b}^{L} s=\Psi^{-1} \square_{b}^{L \otimes K_{X}^{*}} \Psi s .
$$

We can now prove:

Theorem 3.7. With the notations used above, we have on $\Omega^{0, \bullet}(X, L)$,

$$
\begin{aligned}
\square_{b}^{L}= & \Psi^{-1} \bar{\square}_{b}^{L \otimes K_{X}^{*}} \Psi+\left(2(-\sqrt{-1} T) \mathscr{L}+R^{L \otimes K_{X}^{*}}\right)\left(\bar{L}_{j}, L_{k}\right) e_{k} \wedge i_{L_{j}} \\
& +\Psi^{-1}\left(\nabla_{b}^{L \otimes K_{X}^{*}}\right)^{1,0} \mathcal{T}^{*} \Psi-\left[\bar{\partial}_{b}^{L}, \Psi^{-1} \overline{\mathcal{T}}^{*} \Psi\right]
\end{aligned}
$$

where $\left\{\bar{L}_{j}\right\}_{j=1}^{n}$ is a local $\mathbb{R}$-invariant orthonormal frame of $T^{1,0} X$ with dual frame $\left\{\bar{e}_{j}\right\}_{j=1}^{n} \subset T^{* 1,0} X$.

Proof. Let $s \in \Omega^{0, q}(X, L)$. From (3.66) and (1.2), we have

$$
\begin{aligned}
\square_{b}^{L} s= & \Psi^{-1} \square_{b}^{L \otimes K_{X}^{*}} \Psi s \\
= & \Psi^{-1} \bar{\square}_{b}^{L \otimes K_{X}^{*}} \Psi s+\Psi^{-1}[2 \sqrt{-1} \mathscr{L}, \Lambda](-\sqrt{-1} T)(\Psi s) \\
& +\Psi^{-1}\left[\sqrt{-1} R^{L \otimes K_{X}^{*}}, \Lambda\right] \Psi s+\Psi^{-1}\left(\left(\nabla_{b}^{L \otimes K_{X}^{*}}\right)^{1,0} \mathcal{T}^{*}+\mathcal{T}^{*}\left(\nabla_{b}^{L \otimes K_{X}^{*}}\right)^{1,0}\right) \Psi s \\
& -\left(\bar{\partial}_{b}^{L} \Psi^{-1} \overline{\mathcal{T}}^{*} \Psi s+\Psi^{-1} \overline{\mathcal{T}}^{*} \Psi \bar{\partial}_{b}^{L} s\right) .
\end{aligned}
$$

We can check that

$$
\begin{aligned}
{[2 \sqrt{-1} \mathscr{L}, \Lambda] } & =2 \mathscr{L}\left(\bar{L}_{j}, L_{k}\right)\left(\bar{e}_{j} \wedge i_{\bar{L}_{k}}-i_{L_{j}} e_{k} \wedge\right), \\
{\left[2 \sqrt{-1} R^{L \otimes K_{X}^{*}}, \Lambda\right] } & =R^{L \otimes K_{X}^{*}}\left(\bar{L}_{j}, L_{k}\right)\left(\bar{e}_{j} \wedge i_{\bar{L}_{k}}-i_{L_{j}} e_{k} \wedge\right) .
\end{aligned}
$$

From (3.68), (3.69) and notice that

$$
\left(\bar{e}_{j} \wedge i_{\bar{L}_{k}}-i_{L_{j}} e_{k} \wedge\right) v=e_{k} \wedge i_{L_{j}} v
$$


$\mathcal{T}^{*}\left(\nabla^{L \otimes K_{X}^{*}}\right)^{1,0} v=0$, for every $v \in \Omega^{n, q}\left(X, L \otimes K_{X}^{*}\right)$ and $T$ commutes with $\Psi$, we get (3.67).

\section{Two basic vanishing theorems}

Firstly, we present two basic inequalities in this context.

Corollary 4.1 (CR Nakano's inequality I). With the notations used above, for any $s \in \Omega_{c}^{\bullet \bullet \bullet}(X, L)$,

$$
\begin{aligned}
\frac{3}{2}\left(\square_{b}^{L} s \mid s\right) \geq & \left(\left[2 \mathscr{L} T+\sqrt{-1} R^{L}, \Lambda\right] s \mid s\right) \\
& -\frac{1}{2}\left(\|\mathcal{T} s\|^{2}+\left\|\mathcal{T}^{*} s\right\|^{2}+\|\overline{\mathcal{T}} s\|^{2}+\left\|\overline{\mathcal{T}}^{*} s\right\|^{2}\right)
\end{aligned}
$$

If $\left(X, T^{1,0} X\right)$ is Kähler, i.e., $d \Theta_{X}=0$, then

$$
\left(\square_{b}^{L} s \mid s\right) \geq\left(\left[2 \mathscr{L} T+\sqrt{-1} R^{L}, \Lambda\right] s \mid s\right) .
$$

Proof. By CauchySchwarz inequality (also see [13, Theorem 1.4.14]), Theorem 1.2 and note that $\mathcal{T}=0, \mathcal{T}^{*}=0$ if $d \Theta_{X}=0$, we get the corollary.

Similarly, by Theorem 3.7 (also see [13, Corollary 1.4.17]), we have:

Corollary 4.2 (CR Nakano's inequality II). With the notations used above, for any $s \in \Omega_{c}^{0, \bullet}(X, L)$,

$$
\begin{aligned}
\frac{3}{2}\left(\square_{b}^{L} s \mid s\right) \geq & \left(\left(2(-\sqrt{-1} T) \mathscr{L}+R^{L \otimes K_{X}^{*}}\right)\left(\bar{L}_{j}, L_{k}\right) e_{k} \wedge i_{L_{j}} s \mid s\right) \\
& -\frac{1}{2}\left(\left\|\mathcal{T}^{*} \widetilde{s}\right\|^{2}+\|\overline{\mathcal{T}} \widetilde{s}\|^{2}+\left\|\overline{\mathcal{T}}^{*} s\right\|^{2}\right) .
\end{aligned}
$$

If $\left(X, T^{1,0} X\right)$ is Kähler, i.e., $d \Theta_{X}=0$, then

$$
\left(\square_{b}^{L} s \mid s\right) \geq\left(\left(2(-\sqrt{-1} T) \mathscr{L}+R^{L \otimes K_{X}^{*}}\right)\left(\bar{L}_{j}, L_{k}\right) e_{k} \wedge i_{L_{j}} s \mid s\right) .
$$

Remark 4.3. Let $X$ be strictly pseudoconvex CR manifold and $g_{X}$ is the Levi metric such that $2 \sqrt{-1} \mathscr{L}=\Theta_{X}$. Let $\nabla$ be the canonical connection determined by $g_{X}$ and Ric be the pseudohermitian Ricci form. Then, for $s \in \Omega_{0}^{0, q}(X), 1 \leq q \leq n$, it is already known from [15, Theorems 5.1, 5.2] 
that

$$
\left(\square_{b} s \mid s\right) \geq q\left(\nabla_{-\sqrt{-1} T} s \mid s\right)+\left(\operatorname{Ric}\left(\bar{L}_{j}, L_{k}\right) e_{k} \wedge i_{L_{j}} s \mid s\right),
$$

which is compatible to (4.4). It is also an interesting problem to formulate a CR Bochner-KodairaNakano type formula for arbitrary CR manifolds (without group action) as well as to generalize the classical Tanaka's formula [15].

From now on we consider CR manifolds with transversal CR $S^{1}$-action. For $r, q \in \mathbb{N}_{0}, m \in \mathbb{Z}$, we set

$$
\begin{aligned}
\Omega_{m}^{r, q}(X, L) & :=\left\{\Omega_{m}^{r, q}(X, L):-\sqrt{-1} T s=m s\right\} \\
H_{m}^{r, q}(X, L) & :=\operatorname{Ker} \bar{\partial}_{b}^{L} \cap \Omega_{m}^{r, q}(X, L) / \operatorname{Im} \bar{\partial}_{b}^{L} \cap \Omega_{m}^{r, q}(X, L) .
\end{aligned}
$$

From [1, Theorem 3.7] it is known that:

Theorem 4.4 ([1]). Let $X$ be a compact, connected CR manifold of dimension $2 n+1$ with a transversal $C R S^{1}$ action and let $L$ be a rigid $C R$ line bundle over $X$ with a rigid Hermitian metric $h^{L}$ on $L$. Then, for $m \in \mathbb{Z}$,

$$
\operatorname{Ker} \square_{b}^{L} \cap \Omega_{m}^{r, q}(X, L) \cong H_{b, m}^{r, q}(X, L) .
$$

We will need the following simple result by calculation (see [13, Problem $1.10])$.

Proposition 4.5. For a real $(1,1)$-form $\sqrt{-1} \alpha \in \Omega^{1,1}(D)$, if we choose orthonormal frame $\left\{\bar{L}_{j}\right\}_{j=1}^{n}$ of $T^{1,0} D$ with the dual frame $\left\{\bar{e}_{j}\right\}_{j=1}^{n}$ of $T^{* 1,0} D$ such that $\sqrt{-1} \alpha=\sqrt{-1} \lambda_{j}(x) \bar{e}_{j} \wedge e_{j}$ at a given point $x \in D$, then for any $u=\sum_{I, J} u_{I J}(x) \bar{e}^{I} \wedge e^{J} \in \Omega^{\bullet \bullet \bullet}(D)$, we have

$$
[\sqrt{-1} \alpha, \Lambda] u(x)=\sum_{I, J}\left(\sum_{j \in I} \lambda_{j}(x)+\sum_{j \in J} \lambda_{j}(x)-\sum_{j=1}^{n} \lambda_{j}(x)\right) u_{I J}(x) \bar{e}^{I} \wedge e^{J} .
$$

We remark that if $\sqrt{-1} R_{\mathscr{L}}^{L}:=2 \sqrt{-1} \mathscr{L}+\sqrt{-1} R^{L}>0$ on $X$, then it induces a Hermitian metric $\langle\cdot \mid \cdot\rangle_{\mathscr{L}, L}$ on $\mathbb{C} T X$ and $\langle\cdot \mid \cdot\rangle_{\mathscr{L}, L}$ induces a Hermitian metric $\langle\cdot \mid \cdot\rangle_{\mathscr{L}, L}$ on $T^{* \bullet \bullet \bullet} X$. More precisely, we can construct a 
Hermitian metric $\langle\cdot \mid \cdot\rangle_{\mathscr{L}, L}$ on $\mathbb{C} T X=T^{1,0} X \oplus T^{0,1} X \oplus \mathbb{C}\{T\}$ in the following way: For arbitrary $a, b \in T^{1,0} X$, we set

$\langle a \mid b\rangle_{\mathscr{L}, L}:=R_{\mathscr{L}}^{L}(a, \bar{b}),\langle\bar{a} \mid \bar{b}\rangle_{\mathscr{L}, L}:=\langle b \mid a\rangle_{\mathscr{L}, L},\langle a \mid \bar{b}\rangle_{\mathscr{L}, L}:=0,\langle T \mid T\rangle_{\mathscr{L}, L}:=1$.

Similarly, we can consider, for $k, m \in \mathbb{Z}$, the Hermitian metric induced by $2 k \mathscr{L}+\sqrt{-1} R^{L^{m}}>0$.

Proof of Corollary 1.5. Let $s \in \Omega_{m_{0}}^{r, q}(X, L)$. Then

$$
\left[2 \mathscr{L} T+\sqrt{-1} R^{L}, \Lambda\right] s=\left[2 m_{0} \sqrt{-1} \mathscr{L}+\sqrt{-1} R^{L}, \Lambda\right] s .
$$

Let $\Theta_{X}:=2 m_{0} \sqrt{-1} \mathscr{L}+\sqrt{-1} R^{L}$. Then $\Theta_{X}$ is a Kähler metric on $X$. Then the Hermitian torsion $\mathcal{T}=0$. From Corollary 4.1.

$$
\left(\square_{b}^{L} s \mid s\right) \geq\left(\left[\Theta_{X}, \Lambda\right] s \mid s\right)=(r+q-n)\|s\|^{2} \geq\|s\|^{2} .
$$

The proof is complete by Theorem 4.4 .

Proof of Corollary 1.6. Let $s \in \Omega_{m}^{0, q}\left(X, L^{m}\right)$. Then, by Corollary 4.2,

$$
\begin{aligned}
& \left(2(-\sqrt{-1} T) \mathscr{L}+R^{L^{m} \otimes K_{X}^{*}}\right)\left(\bar{L}_{j}, L_{k}\right) e_{k} \wedge i_{L_{j}} s \\
= & m\left(2 \mathscr{L}+R^{L}\right)\left(\bar{L}_{j}, L_{k}\right) e_{k} \wedge i_{L_{j}} s+R^{K_{X}^{*}}\left(\bar{L}_{j}, L_{k}\right) e_{k} \wedge i_{L_{j}} s
\end{aligned}
$$

Let $\Theta_{X}:=2 \sqrt{-1} \mathscr{L}+\sqrt{-1} R^{L}$. Then $\Theta_{X}$ is a Kähler metric on $X$ and there exists $C>0$ such that

$$
\left(\square_{b}^{L^{m}} s \mid s\right) \geq(m q-C)\|s\|^{2} \geq(m-C)\|s\|^{2}
$$

for $m>0$. The proof is complete by Theorem 4.4 .

\section{Further Results and Application}

Let $X$ be a CR manifold with a transversal CR $\mathbb{R}$-action. Let $\Theta_{X}$ be an $\mathbb{R}$-invariant Hermitian metric on $X$. Let $g_{X}$ be the underlying Riemannian metric associated to $\Theta_{X}$ on $X$. Let $L$ and $F$ be a $\mathbb{R}$-equivariant CR line bundle over $X$ with a $\mathbb{R}$-invariant Hermitian metric $h^{L}$ and $h^{F}$.

The main goal of $\mathrm{CR}$ vanishing theorems is to find natural geometric condition on the curvature of $L$ or the Levi form $\mathscr{L}$ on $X$ such that Kohn- 
Rossi cohomology group or the space of harmonic forms with values in $L$ vanish. In this section, we show several refinement of Corollary 1.5 and 1.6 analogue to the complex manifold case. This section follows closely from [3, Ch. VII. Sec. 2-Sec. 5].

\subsection{Basic priori inequality}

Let $X$ be compact with $\operatorname{dim} X=2 n+1$. For $s \in \Omega^{p, q}(X, L)$, we have $\left(\square_{b}^{L} s \mid s\right)=\left\|\bar{\partial}_{b}^{L} s\right\|^{2}+\left\|\bar{\partial}_{b}^{L *} s\right\|^{2} \geq 0$ and the similar formula for $\bar{\square}_{b, \mathcal{T}}^{L}$ shows that $\left(\bar{\square}_{b, \mathcal{T}}^{L} s \mid s\right) \geq 0$. Theorem 1.3 .

$$
\square_{b}^{L}=\square_{b, \mathcal{T}}^{L}+\left[2 \mathscr{L} T+\sqrt{-1} R^{L}, \Lambda\right]+\mathcal{T}_{\Theta},
$$

gives

$$
\begin{aligned}
\left\|\bar{\partial}_{b}^{L} s\right\|^{2}+\left\|\bar{\partial}_{b}^{L *} s\right\|^{2} & =\left(\bar{\square}_{b, \mathcal{T}}^{L} s \mid s\right)+\int_{X}\left\langle\left[2 \mathscr{L} T+\sqrt{-1} R^{L}, \Lambda\right] s \mid s\right\rangle+\left\langle\mathcal{T}_{\Theta} s \mid s\right\rangle d v_{X} \\
& \geq \int_{X}\left\langle\left[2 \mathscr{L} T+\sqrt{-1} R^{L}, \Lambda\right] s \mid s\right\rangle+\left\langle\mathcal{T}_{\Theta} s \mid s\right\rangle d v_{X} .
\end{aligned}
$$

If $\square_{b}^{L} s=0$, we obtain

$$
0 \geq \int_{X}\left\langle\left[2 \mathscr{L} T+\sqrt{-1} R^{L}, \Lambda\right] s \mid s\right\rangle+\left\langle\mathcal{T}_{\Theta} s \mid s\right\rangle d v_{X}
$$

Note that $\left[2 \mathscr{L} T+\sqrt{-1} R^{L}, \Lambda\right]+\mathcal{T}_{\Theta}=\square_{b}^{L}-\bar{\square}_{b, \mathcal{T}}^{L}$ is formally self-adjoint on $\Omega^{p, q}(X, L)$. Thus we have:

Proposition 5.1. Let $X$ be compact. If $0 \leq p, q \leq n$ and $([2 \mathscr{L} T+$ $\left.\left.\sqrt{-1} R^{L}, \Lambda\right]+\mathcal{T}_{\Theta} s \mid s\right)>0$ for all $s \in \Omega^{p, q}(X, L)$, then

$$
\operatorname{Ker} \square_{b}^{L} \cap \Omega^{p, q}(X, L)=0 .
$$

\subsection{CR Akizuki-Nakano vanishing theorem}

Let $\square_{b}^{L}$ be the Gaffney extension of the usual Kohn Laplacian in this section.

Theorem 5.2. Let $\mathscr{L}=0$ on $X$. 
(a) If $\sqrt{-1} R^{L}$ is positive and $\Theta_{X}=\sqrt{-1} R^{L}$ is complete, then, for $p+q \geq$ $n+1$,

$$
\operatorname{Ker} \square_{b}^{L} \cap L_{p, q}^{2}(X, L)=0 .
$$

(b) If $\sqrt{-1} R^{L}$ is negative and $\Theta_{X}=-\sqrt{-1} R^{L}$ is complete, then, for $p+q \leq$ $n-1$,

$$
\operatorname{Ker} \square_{b}^{L} \cap L_{p, q}^{2}(X, L)=0 .
$$

Proof. In the case (a), we have $\Theta_{X}=\sqrt{-1} R^{L}$ is complete Kähler metric on $X$. Then, for $s \in \Omega_{c}^{p, q}(X, L)$ with $p+q \geq n+1$, we have

$$
\left(\left[\sqrt{-1} R^{L}, \Lambda\right] s \mid s\right)=(p+q-n)\|s\|^{2} \geq\|s\|^{2},
$$

and thus $\left\|\bar{\partial}_{b}^{L} s\right\|^{2}+\left\|\bar{\partial}_{b}^{L *} s\right\|^{2} \geq\|s\|^{2}$. By the density lemma, the previous inequality holds for $s \in \operatorname{Dom} \bar{\partial}_{b}^{L} \cap \operatorname{Dom} \bar{\partial}_{b}^{L *} \cap L_{p, q}^{2}(X, L)$. In particular, the assertion (a) holds.

In the case (b), we have $\Theta_{X}=-\sqrt{-1} R^{L}$ is complete Kähler metric on $X$. Then, for $s \in \Omega_{c}^{p, q}(X, L)$ with $p+q \leq n-1$, we have

$$
\left(\left[\sqrt{-1} R^{L}, \Lambda\right] s \mid s\right)=(n-(p+q))\|s\|^{2} \geq\|s\|^{2},
$$

and thus $\left\|\bar{\partial}_{b}^{L} s\right\|^{2}+\left\|\bar{\partial}_{b}^{L *} s\right\|^{2} \geq\|s\|^{2}$. By the density lemma, the previous inequality holds for $s \in \operatorname{Dom} \bar{\partial}_{b}^{L} \cap \operatorname{Dom} \bar{\partial}_{b}^{L *} \cap L_{p, q}^{2}(X, L)$. In particular, the assertion (b) holds.

From Theorem 4.4, we have a immediate consequence as follows, which can be viewed as CR Akizuki-Nakano vanishing theorem.

Corollary 5.3. Let $X$ be a compact $C R$ manifold with a transversal $C R S^{1}$ action. Let $L$ be a $S^{1}$-equivariant $C R$ line bundle over $X$ with a $S^{1}$-invariant Hermitian metric $h^{L}$. Let $\mathscr{L}=0$ on $X$.

(a) If $\sqrt{-1} R^{L}$ is positive, then, for $p+q \geq n+1$, we have

$$
H_{b, m}^{p, q}(X, L)=0, \quad m \in \mathbb{Z} .
$$

(b) If $\sqrt{-1} R^{L}$ is negative, then, for $p+q \leq n-1$, we have

$$
H_{b, m}^{p, q}(X, L)=0, \quad m \in \mathbb{Z} .
$$


We remark that, instead of the case of trivial Levi form $\mathscr{L}=0$, the case of trivial line bundle $L=X \times \mathbb{C}$ was considered in [9], see the notions and proof therein. We obtained a CR counterpart of the Kodaira vanishing theorem as follows.

Corollary $5.4([9])$. Assume that $2 \sqrt{-1} \mathscr{L}=\Theta_{X}, g_{X}$ is complete and let $\lambda<0$ and $1 \leq q \leq n$. Then, we have

$$
\operatorname{Ker} \square_{b} \cap \mathscr{E}(\lambda, \sqrt{-1} T) \cap L_{n, q}^{2}(X)=0 .
$$

Also we obtained a CR counterpart of the Kodaira-Serre vanishing theorem as follows.

Corollary $5.5([9])$. Assume that $2 \sqrt{-1} \mathscr{L}=\Theta_{X}, g_{X}$ is complete and let $C \geq 0$ such that

$$
2 \sqrt{-1} \mathscr{L}=\Theta_{X}, \quad \sqrt{-1} R^{K_{X}^{*}} \geq-C \Theta_{X}
$$

Let $\lambda<-C$ and $1 \leq q \leq n$. Then, we have

$$
\operatorname{Ker} \square_{b} \cap \mathscr{E}(\lambda, \sqrt{-1} T) \cap L_{0, q}^{2}(X)=0 .
$$

\subsection{Girbau's vanishing theorem in the CR setting}

Assume that $X$ is compact, $d \Theta_{X}=0$ (i.e., $\Theta_{X}$ is Kähler metric) and $\mathscr{L}=0$ on $X$ in this section, we will use the formula

$$
\square_{b}^{L}=\bar{\square}_{b}^{L}+\left[\sqrt{-1} R^{L}, \Lambda\right] .
$$

to obtain the analogue of Girbau's theorem with degenerate semi-positive line bundle in the CR setting.

Theorem 5.6. Let $X$ be compact. Let $\mathscr{L}=0$ and $d \Theta_{X}=0$. If $\sqrt{-1} R^{L}$ is semi-positive and has at least $n-s+1$ positive eigenvalues at every point $x \in X$, then there exists a Kähler metric $\Theta_{X, \epsilon}$ on $X$ such that

$$
\operatorname{Ker} \square_{b, \epsilon}^{L} \cap \Omega^{p, q}(X, L)=0, \quad p+q \geq n+s,
$$

where $\square_{b, \epsilon}^{L}$ is with respect to $\Theta_{X, \epsilon}$ and $h^{L}$. 
Proof. Let us construct a new Kähler metric

$$
\Theta_{X, \epsilon}=\epsilon \Theta_{X}+\sqrt{-1} R^{L}, \quad \epsilon>0 .
$$

Here $\epsilon$ will be determined later. Let $\sqrt{-1} R^{L}=\sqrt{-1} \sum_{j=1}^{n} \lambda_{j} \bar{e}_{j} \wedge e_{j}$ with $\lambda_{1} \leq \cdots \leq \lambda_{n}$ as in Proposition 4.5. Then

$$
\Theta_{X, \epsilon}=\sqrt{-1} \sum_{j=1}^{n}\left(\epsilon+\lambda_{j}\right) \bar{e}_{j} \wedge e_{j} .
$$

Thus the eigenvalues of $\sqrt{-1} R^{L}$ with respect to $\Theta_{X, \epsilon}$ are given by

$$
\lambda_{j, \epsilon}=\frac{\lambda_{j}}{\epsilon+\lambda_{j}} \in[0,1), \quad 1 \leq j \leq n .
$$

The hypothesis implies that $\lambda_{s}(x)>0$ at every point $x$ of $X$. For $j \geq s$ we have $\lambda_{j} \geq \lambda_{s}$, thus

$$
\lambda_{j, \epsilon}=\frac{1}{1+\frac{\epsilon}{\lambda_{j}}} \geq \frac{1}{1+\frac{\epsilon}{\lambda_{s}}} \geq 1-\frac{\epsilon}{\lambda_{s}}, \quad s \leq j \leq n .
$$

Let us denote the operator and Hermitian inner product with respect to $\Theta_{X, \epsilon}$ with $\epsilon$ as an index. For $s \in \Omega^{p, q}(X, L)$ with $p+q \geq n+s$, we have $q-s \geq n-p \geq 0$ and $p-s \geq n-q \geq 0$. By Proposition 4.5, locally we have

$$
\begin{aligned}
\left\langle\left[\sqrt{-1} R^{L}, \Lambda_{\epsilon}\right] s \mid s\right\rangle_{\epsilon} & =\sum_{I, J}\left(\sum_{j \in I} \lambda_{j}(x)+\sum_{j \in J} \lambda_{j}(x)-\sum_{j=1}^{n} \lambda_{j}(x)\right)\left|s_{I J}(x)\right|^{2} \\
& \geq\left(\sum_{j=1}^{q} \lambda_{j, \epsilon}-\sum_{j=p+1}^{n} \lambda_{j, \epsilon}\right)|s|_{\epsilon}^{2} \\
& \geq\left(\sum_{j=1}^{s-1} \lambda_{j, \epsilon}+\sum_{j=s}^{q} \lambda_{j, \epsilon}-\sum_{j=p+1}^{n} \lambda_{j, \epsilon}\right)|s|_{\epsilon}^{2} \\
& \geq\left((q-s+1)\left(1-\frac{\epsilon}{\lambda_{s}}\right)-(n-p)\right)|s|_{\epsilon}^{2} \\
& \geq\left(p+q-n-s+1-(q-s+1) \frac{\epsilon}{\lambda_{s}}\right)|s|_{\epsilon}^{2} .
\end{aligned}
$$


Finally we choose $\epsilon>0$ such that

$$
\epsilon<\frac{p+q-n-s+1}{q-s+1} \min _{x \in X} \lambda_{s}(x),
$$

where $\min _{x \in X} \lambda_{s}(x)$ can be achieved by the compactness of $X$. The proof is complete by applying

$$
\square_{b, \epsilon}^{L}=\square_{b, \epsilon}^{L}+\left[\sqrt{-1} R^{L}, \Lambda_{\epsilon}\right]
$$

on $s \in \Omega^{p, q}(X, L)$ with $p+q \geq n+s$.

From Theorem 4.4, we have

Corollary 5.7. Let $X$ be a compact $C R$ manifold with a transversal $C R S^{1}$ action. Let $\mathscr{L}=0$ and $d \Theta_{X}=0$. Let $L$ be a $S^{1}$-equivariant $C R$ line bundle over $X$ with a $S^{1}$-invariant Hermitian metric $h^{L}$. If $\sqrt{-1} R^{L}$ is semi-positive and has at least $n-s+1$ positive eigenvalues at every point $x \in X$, then

$$
H_{b, m}^{p, q}(X, L)=0, \quad p+q \geq n+s, m \in \mathbb{Z} .
$$

\subsection{Vanishing theorem for partially positive CR line bundles}

Even in the case that $\sqrt{-1} R^{L}$ is not semi-positive, Kohn-Rossi cohomology (or the space of harmonic form) of high tensor powers $L^{k}$ still vanish under suitable assumptions. The following can be viewed as a analogue result of Andreotti-Grauert theorem in the CR setting.

Theorem 5.8. Let $X$ be compact. Let $\mathscr{L}=0$. Let $1 \leq s \leq n$ and $\sqrt{-1} R^{L}$ has at least $n-s+1$ positive eigenvalues at every point $x \in X$. Then there exist $k_{0} \in \mathbb{N}$ and a Hermitian metric $\Theta_{X, \epsilon}$ such that

$$
\operatorname{Ker} \square_{b, \epsilon}^{L^{k} \otimes F} \cap \Omega^{n, q}\left(X, L^{k} \otimes F\right)=0, \quad k \geq k_{0}, q \geq s>0,
$$

where $\square_{b, \epsilon}^{L^{k} \otimes F}$ is with respect to $\Theta_{X, \epsilon}, h^{L}$ and $h^{F}$.

Proof. The idea is to construct a Hermitian metric $\Theta_{X, \epsilon}$ on $X$ such that all negative eigenvalues of $\sqrt{-1} R^{L}$ with respect to $\Theta_{X, \epsilon}$ will be of sufficiently small absolute value.

We will use the fact: 
Lemma 5.3 (3] , Ch. VII. (5.2)). Let $\psi \in \mathscr{C}^{\infty}(\mathbb{R}, \mathbb{R})$. If $A$ is a Hermitian matrix with eigenvalues $\lambda_{1} \leq \cdots \leq \lambda_{n}$ and corresponding eigenvectors $v_{1}, \cdots, v_{n}$, we define $\psi(A)$ as the Hermitian matrix with eigenvalues $\psi\left(\lambda_{j}\right)$ with eigenvectors $v_{1}, \cdots, v_{n}$. Then, the map $\psi: A \rightarrow \psi(A)$ is smooth on $\operatorname{Herm}\left(\mathbb{C}^{n}\right)$.

Let $\Theta_{X}$ be the fixed Hermitian metric on $X$ and let $\lambda_{1} \leq \cdots \leq \lambda_{n}$ be the corresponding eigenvalues of $\sqrt{-1} R^{L}$ given by locally $\sqrt{-1} R^{L}=$ $\sqrt{-1} \sum_{j} \lambda_{j} \bar{e}_{j} \wedge e_{j}$. Note that $\lambda_{s}>0$ by the hypothesis on $\sqrt{-1} R^{L}$.

Let

$$
t_{0}=\inf _{X} \lambda_{s}>0, \quad M=\sup _{X} \max _{1 \leq j \leq n}\left|\lambda_{j}\right|>0 .
$$

Let $\epsilon>0$, which will be determined later. We can choose $\psi_{\epsilon} \in \mathscr{C}^{\infty}(\mathbb{R}, \mathbb{R})$ such that

$$
\begin{aligned}
& \psi_{\epsilon}(t)=t, \quad t \geq t_{0} \\
& \psi_{\epsilon}(t) \geq t, \quad 0<t \leq t_{0} \\
& \psi_{\epsilon}(t)=\frac{M}{\epsilon}, \quad t \leq 0
\end{aligned}
$$

By applying the above Lemma to $\psi_{\epsilon}$,

$$
\Theta_{X, \epsilon}:=\psi_{\epsilon}\left(\sqrt{-1} R^{L}\right)
$$

is a smooth Hermitian metric on $X$. We write

$$
\sqrt{-1} R^{L}=\sqrt{-1} \sum_{j=1}^{n} \lambda_{j} \bar{e}_{j} \wedge e_{j}, \quad \Theta_{X, \epsilon}=\sqrt{-1} \sum_{j=1}^{n} \psi_{\epsilon}\left(\lambda_{j}\right) \bar{e}_{j} \wedge e_{j},
$$

where $\left\{\bar{e}_{j}\right\}_{j=1}$ are local orthonormal frame of $T^{1,0 *} X$ with respect to $\Theta_{X}$. The eigenvalues of $\sqrt{-1} R^{L}$ with respect to $\Theta_{X, \epsilon}$ are given by

$$
\lambda_{j, \epsilon}=\frac{\lambda_{j}}{\psi_{\epsilon}\left(\lambda_{j}\right)}
$$

The construction of $\psi_{\epsilon}$ shows that

$$
\begin{aligned}
-\epsilon \leq \lambda_{j, \epsilon} \leq 1, & \text { for } 1 \leq j \leq n . \\
\lambda_{j, \epsilon}=1, & \text { for } s \leq j \leq n .
\end{aligned}
$$


In fact, we have

$$
\begin{aligned}
& \lambda_{j, \epsilon}=1, \quad \text { when } \lambda_{j} \geq t_{0} \\
& \lambda_{j, \epsilon} \leq 1, \quad \text { when } 0<\lambda_{j} \leq t_{0} \\
& \lambda_{j, \epsilon}=\epsilon \frac{\lambda_{j}}{M} \geq-\epsilon, \quad \text { when } \lambda_{j} \leq 0,
\end{aligned}
$$

and if $s \leq j \leq n$, then $\lambda_{j} \geq \lambda_{s} \geq t_{0}$ and thus $\lambda_{j, \epsilon}=1$.

Now let $s \in \Omega^{n, q}\left(X, L^{k} \otimes F\right)$. Locally we write

$$
s=\sum_{|J|=q} s_{J} \bar{e}_{N} \wedge e_{J} \otimes e_{L}^{k} \otimes f
$$

where $e_{L}$ and $f$ denote local orthonormal frame of $L$ and $F$. Then, note $q \geq s$, we have

$$
\begin{aligned}
\left\langle\left[\sqrt{-1} R^{L}, \Lambda_{\epsilon}\right] s \mid s\right\rangle_{\epsilon} & =\sum_{J}\left(\sum_{j \in J} \lambda_{j, \epsilon}\right)\left|s_{J}\right|^{2} \\
& \geq((q-s+1)-(s-1) \epsilon)|s|_{\epsilon}^{2} .
\end{aligned}
$$

Choose $\epsilon=\frac{1}{s}$ and thus

$$
\left\langle\left[\sqrt{-1} R^{L}, \Lambda_{\epsilon}\right] s \mid s\right\rangle_{\epsilon} \geq \frac{1}{s}|s|_{\epsilon}^{2} .
$$

Since that $R^{L^{k} \otimes F}=k R^{L}+R^{F}$, there exist $k_{0}>0$ and $C>0$ such that for $k \geq k_{0}$, we have

$$
\left(\square_{b, \epsilon}^{L^{k} \otimes F} s \mid s\right)_{\epsilon} \geq\left(\left[\sqrt{-1} R^{L^{k} \otimes F}, \Lambda_{\epsilon}\right] s \mid s\right)_{\epsilon}+\left(\mathcal{T}_{\Theta_{X, \epsilon}} s \mid s\right)_{\epsilon} \geq C\|s\|_{\epsilon}^{2} .
$$

The proof is complete by Theorem 1.3 .

From Theorem 4.4, we have

Corollary 5.9. Let $X$ be a compact $C R$ manifold with a transversal $C R$ $S^{1}$-action. Let $L, F$ be a $S^{1}$-equivariant $C R$ line bundle over $X$ with a $S^{1}$ invariant Hermitian metric $h^{L}, h^{F}$. Let $\mathscr{L}=0$. Let $1 \leq s \leq n$ and $\sqrt{-1} R^{L}$ has at least $n-s+1$ positive eigenvalues at every point $x \in X$. Then there exists $k_{0}>0$ such that

$$
H_{b, m}^{n, q}\left(X, L^{k} \otimes F\right)=0, \quad k \geq k_{0}, q \geq s>0, m \in \mathbb{Z} .
$$


Remark 5.10. By replacing $F$ by $F \otimes \operatorname{det}\left(T^{1,0} X\right)$ above, we also have

$$
H_{b, m}^{0, q}\left(X, L^{k} \otimes F\right)=0, \quad k \geq k_{0}, q \geq s>0, m \in \mathbb{Z} .
$$

\subsection{On the CR index theorem with $S^{1}$ action}

Many classical results in algebraic and complex geometry are derived by combining a cohomology vanishing theorem with an index theorem. Follow this principal, We combine the the CR index theorem with $S^{1}$ and Corollary 1.5 and [1.6 to detect the existence of CR sections, see [1, Corollary 1.13, Corollary 1.20].

Theorem 5.11 ([1] ). Let $X$ be a compact, connected $C R$ manifold of dimension $2 n+1$ with a transversal $C R$ locally free $S^{1}$ action and let $E$ be a rigid CR vector bundle over $X$. Then, for $m \in \mathbb{Z}$,

$$
\begin{aligned}
& \sum_{j=0}^{n}(-1)^{j} \operatorname{dim} H_{b, m}^{j}(X, E) \\
& =p \delta_{p \mid m} \frac{1}{2 \pi} \int_{X} \operatorname{Td}_{b}\left(T^{1,0 X}\right) \wedge \operatorname{ch}_{b}(E) \wedge e^{m \frac{d \omega_{0}}{2 \pi}} \wedge \omega_{0} \\
& =\operatorname{rank}(E) p \delta_{p \mid m} \frac{m^{n}}{n !(2 \pi)^{n+1}} \int_{X}\left(d \omega_{0}\right)^{n} \wedge \omega_{0}+O\left(m^{n-1}\right),
\end{aligned}
$$

where $\operatorname{Td}_{b}\left(T^{1,0 X}\right)$ denotes the tangential Todd class of $T^{1,0} X$ and $\operatorname{ch}_{b}(E)$ denotes the tangential Chern character of $E$.

Therefore, from Corollary 1.5 and Corollary 1.6, it follows that:

Corollary 5.12. Let $X$ be a compact, connected $C R$ manifold of dimension $2 n+1$ with a transversal $C R$ locally free $S^{1}$ action and let $L$ be a rigid $C R$ line bundle over $X$ with a rigid Hermitian metric $h^{L}$ on $L$.

(1) If $m_{0} \in \mathbb{Z}$ such that $2 m_{0} \sqrt{-1} \mathscr{L}+\sqrt{-1} R^{L}>0$ on $X$, then we have

$$
\begin{aligned}
& \operatorname{dim} H_{b, m_{0}}^{0}\left(X, K_{X} \otimes L\right) \\
& =p \delta_{p \mid m_{0}} \frac{1}{2 \pi} \int_{X} T d_{b}\left(T^{1,0} X\right) \wedge \operatorname{ch}_{b}\left(K_{X} \otimes L\right) \wedge e^{m_{0} \frac{d \omega_{0}}{2 \pi}} \wedge \omega_{0} \\
& =p \delta_{p \mid m_{0}} \frac{m_{0}^{n}}{n !(2 \pi)^{n+1}} \int_{X}\left(d \omega_{0}\right)^{n} \wedge \omega_{0}+O\left(m_{0}^{n-1}\right) .
\end{aligned}
$$


(2) If $2 \sqrt{-1} \mathscr{L}+\sqrt{-1} R^{L}>0$ on $X$, then there exists $m_{0}>0$ such that for each $m>m_{0}$, we have

$$
\begin{aligned}
\operatorname{dim} H_{b, m}^{0}\left(X, L^{m}\right) & =p \delta_{p \mid m} \frac{1}{2 \pi} \int_{X} T d_{b}\left(T^{1,0} X\right) \wedge \operatorname{ch}_{b}\left(L^{m}\right) \wedge e^{m \frac{d \omega_{0}}{2 \pi}} \wedge \omega_{0} \\
& =p \delta_{p \mid m} \frac{m^{n}}{n !(2 \pi)^{n+1}} \int_{X}\left(d \omega_{0}\right)^{n} \wedge \omega_{0}+O\left(m^{n-1}\right) .
\end{aligned}
$$

\section{Acknowledgments}

The author thanks Professors Guokuan Shao and Xiaoshan Li for their financial support. He also thanks his Postdoc friends, Jian Zhang, Bingying Lu, Hao Qi, Yishuo Shi, Ji Guo and Weixia Zhu for their help during the Coronavirus pandemic. In particular, the author wishes to express his hearty thanks to Professor Chunle Huang and Professor Yong Huang from Hunan University for their enthusiasm.

\section{References}

1. J.-H. Cheng, C.-Y. Hsiao and I-H. Tsai, Heat kernel asymptotics, local index theorem and trace integrals for CR manifolds with $S^{1}$ action, Mém. Soc. Math. Fr., 162(2019), $1-139$.

2. J.-P. Demailly, Sur l'identité de Bochner-Kodaira-Nakano en géométrie hermitienne, Lecture Notes in Math., 1198 (1985), 88-97, Springer Verlag.

3. J.-P. Demailly, Complex analytic and differential geometry, 2012, published online at www-fourier.ujf-grenoble.fr/ demailly/manuscripts/agbook.pdf.

4. C.-Y. Hsiao, Szegő kernel asymptotics for high power of CR line bundles and Kodaira embedding theorems on CR manifolds, Mem. Amer. Math. Soc., 254 (2018), no. 1217.

5. H. Herrmann, C.-Y. Hsiao and X. Li, Szegő kernels and equivariant embedding theorems for CR manifolds, arXiv:1710.04910, to appear in Math. Res. Lett.

6. H. Herrmann and X. Li, Morse inequalities and embeddings for CR manifolds with circle action, Bull. Inst. Math. Acad. Sin. (N.S.), 15 (2020), No.2, 93-122.

7. C.-Y. Hsiao and G. Marinescu, On the singularities of the Szegö projections on lower energy forms, J. Diff. Geom., 107 (2017), 83-155.

8. C.-Y. Hsiao, G. Marinescu and X. Li, Equivariant Kodaira embedding for CR manifolds with circle action, Michigan Math. J., 70 (2021), No.1, 55-113.

9. C.-Y. Hsiao, G. Marinescu and H. Wang, Szegő kernels asymptotics on some noncompact complete CR manifolds, arXiv:2012.11457v1. 
10. C.-Y. Hsiao and W.-C. Shen, On the second coefficient of the asymptotic expansion of Boutet de Monvel-Sjöstrand, Bull. Inst. Math. Acad. Sin. (N.S.), 15(2020), No. 4, 329-365.

11. R.-T. Huang, The anomaly formula of the analytic torsion on CR manifolds with $S^{1}$ action, Bull. Inst. Math. Acad. Sin. (N.S.), 12 (2017), No. 2, 153-203.

12. R.-T. Huang and G. Shao, The asymptotics of the $L^{2}$-analytic torsion on CR manifolds with $S^{1}$ action, Bull. Inst. Math. Acad. Sin. (N.S.), 14 (2019), No. 4, 461-491.

13. X. Ma and G. Marinescu, Holomorphic Morse inequalities and Bergman kernels, Progress in Math., Vol. 254, Birkhäuser Verlag, Basel, 2007.

14. W.-C. Shen, Asymptotics of torus equivariant Szegö kernel on a compact CR manifold, Bull. Inst. Math. Acad. Sin. (N.S.), 14 (2019), No. 3, 331-383.

15. N. Tanaka, A differential geometric study on strictly pseudo-convex manifolds, Kinokuniya Book-Store Co. Ltd., Tokyo, 1975, Lectures in Mathematics, Department of Mathematics, Kyoto University, No. 9. 